\title{
THE CONJUGACY PROBLEM FOR GROUPS OF ALTERNATING PRIME TAME LINKS IS POLYNOMIAL-TIME
}

\author{
KARIN JOHNSGARD
}

\begin{abstract}
An alternating projection of a prime link can to used to produce a group presentation (of the link group under free product with the infinite cyclic group) with some useful peculiarities, including small cancellation conditions. In this presentation, conjugacy diagrams are shown to have the form of a tiling of squares in the Euclidean plane in one of a limited number of shapes. An argument based on the shape of the link projection is used to show that the tiling requires no more square tiles than a linear function of word length (with constant multiple based on the number of link crossings). It follows that the computation time for testing conjugacy of two group elements (previously known to be no worse than exponential) is bounded by a cubic polynomial. This bounds complexity in the original link group.
\end{abstract}

\section{INTRODUCTION}

1A. History. The earliest method for finding a presentation of the group of a (tame) link was described by Wirtinger, but Dehn in 1911 published quite a different method of giving a presentation of the link group, and gave knot-theoretic motivation for the decision problems he then posed. One of these decision problems was the Conjugacy Problem: Find an algorithm which decides, given two arbitrary words $w$ and $u$ in the group generators, whether there exists a word $c$ such that the concatenated word $c u c^{-1}$ represents the same group element as $w$. Dehn stated the knot-theoretic motivation for examining the Conjugacy Problem this way: "Two closed curves in the complement of a knot can be deformed continuously into each other without penetrating the knot if and only if the corresponding elements of the knot group are conjugate." (Cf. Stillwell's translation [D, p. 134].)

Dehn's presentation of the link group may be modified to prove that for links with projections of a certain type, the free product of the link group with the infinite cyclic group has a particularly symmetric presentation satisfying the small cancellation conditions $\mathrm{C}^{\prime \prime}(4)$ and $\mathrm{T}(4)$ [We]. (Roughly speaking, these conditions state that the relators listed in the group presentation may be thought of as squares, in some 2-complex of nonpositive curvature.) These small cancellation conditions imply that the Conjugacy Problem for the link group itself is solvable, and requires no worse than exponential time [Scp]. This method has been extended to solve the Conjugacy Problem for the group of any alternating knot [AS1]. A very different approach has recently been used for the Conjugacy Problem for all knots [Se]; this approach does not impose a bound on the computation time.

Received by the editors May 26, 1994 and, in revised form, September 11, 1995.

1991 Mathematics Subject Classification. Primary 20F10, 03D15, 57M25.

The author was supported in part by Alfred P. Sloan Dissertation Fellowship Grant \#DD-414. 
In this paper we consider groups of alternating prime links, using the same approach as in [We]. We use a topological and geometric approach to derive a cubictime solution for the Conjugacy Problem. This is accomplished using a tiling of "relator squares" in the Euclidean plane. The algorithm depends upon the fact that there is a natural "parity" on the group generators (which is induced by checkering in contrasting colors the regions formed in the plane by the link projection), on the small cancellation properties, and on the fact that all of the relators alternate in sign. The bound on the algorithm for checking conjugacy uses directly the origin of the group presentation in the geometry of the link diagram.

It may be added that for knots which are alternating but not prime, the knot group under free product with the infinite cyclic group has a presentation of a special small cancellation type involving half-squares [GeS2]. The same conjugacy algorithm in this case requires exponential time.

I would like to thank Paul Schupp and Ken Appel, who patiently endured earlier versions ad nauseam, and also Mary-Elizabeth Hamstrom, Dick Bishop, Stephanie Alexander, and Wolfgang Haken. I am also very grateful to the referee, whose suggestions made the text much more comprehensible.

1B. Summary and outline of the argument. It has been necessary in this summary to use some technical terms, and for clarity some brief indication has been made of what each of these mean. Rigorous definitions are contained in the main body of the text.

Throughout the following discussion, "diagram" means "simply connected, reduced small cancellation diagram of the presentation described." The word "disk" means "diagram with at least one 2-cell and no cut vertices" (a cut vertex is one whose removal causes a diagram to be disconnected). A chain is a disk which is a $1 \times n$ rectangle of 2 -cells (see section $3 \mathrm{~A}$, Figure 2 ); to say that a chain occurs on the boundary of a disk means that (at least) one long side of the chain and both short ones lie in the boundary of the disk. An $(n+2)$-length word labelling three consecutive sides of a chain is called a chain word.

Suppose that a presentation satisfies $\mathrm{C}^{\prime \prime}(4)$ and $\mathrm{T}(4)$, and that all of the relators are cyclically freely reduced and of length exactly four. If a disk has exactly four chains on its boundary, then it can be placed in the Euclidean plane with all 2-cells actual squares and its boundary non-self-intersecting [J2].

A word in a $\mathrm{C}^{\prime \prime}(4)-\mathrm{T}(4)$ presentation is a geodesic (shortest length representative) if and only if it is freely reduced and contains no chain subwords [AS2], [GeS1], [K], $[\mathrm{J} 2]$.

In a $\mathrm{C}^{\prime \prime}(4)-\mathrm{T}(4)$ presentation with all relators of length four, diagrams which are tilings of squares in the Euclidean plane are uniquely determined, within the confines of a rectangular boundary, by the label of any geodesic edge path of maximal length in the diagram [J2]. (We will give a more precise statement later.)

A word is a cyclic geodesic if all cyclic permutations are geodesics. Two words are cyclically equivalent if some cyclic permutation of one word equals some cyclic permutation of the other.

Observation. Let a group presentation be such that all of the defining relators are even in length and alternating in sign. Then the label of any edge path in a disk is alternating in sign. If two cyclic geodesics are conjugate but not cyclically equivalent, then they are each even in length and alternating in sign. 
We shall say that a group presentation which is as described in the above observation is alternating. For any group with a finite presentation, the free product of the group with the infinite cyclic group has an alternating presentation (see section $3 \mathrm{C})$.

If words $u$ and $c^{-1} w c$ are equal (i.e., represent the same group element), let a conjugacy diagram for $u$ and $w$ (and the conjugating word $c$ ) be a (simply connected) diagram with boundary cycle label $c^{-1} w c u^{-1}$.

For an arbitrary group presentation (with cyclically freely reduced relators): if $u$ and $w$ are cyclic geodesics which are conjugate but not cyclically equivalent, then for some cyclic permutation $\tilde{u}$ of $u$ and some cyclic permutation $\tilde{w}$ of $w$, there is a conjugacy diagram for $\tilde{w}$ and $\tilde{u}$ which is a disk. The conjugating word for this disk may be taken to be of minimal length. (See [J1].)

We say that a presentation has parity if each generator pair $x_{i}^{ \pm}$may be assigned a color black or white so that the letters of any defining relator are alternately black and white.

Theorem 1. Suppose that a group presentation has parity, satisfies $C^{\prime \prime}(4)$ and $T(4)$, and has only relators of length four. Let $u$ and $w$ be cyclic geodesics which are conjugate but not cyclically equivalent.

There is a conjugacy diagram for cyclic permutations $\tilde{u}$ and $\tilde{w}$ of $u$ and $w$ which is a disk. Any such disk has exactly four chains on its boundary, arranged in one of a limited number of possible configurations. This disk may be interpreted as a square tiling in the Euclidean plane, in which depiction the edge paths labelled by the conjugating word are identical under linear translation, and therefore the edge paths labelled by $\tilde{u}$ and $\tilde{w}$ have identical total displacements. It follows that the number of letters of $w$ of black parity is the same as the number of letters of $u$ of black parity, and likewise for white.

Remark. Theorem 1 implies that in this class of presentations, a word is a shortest representative of its conjugacy class if and only if it is a cyclic geodesic. This is false without the parity assumption. However, Mile Krajcevski and I have recently characterized shortest conjugacy representatives for $\mathrm{C}^{\prime \prime}(p)-\mathrm{T}(q)$ presentations where all relators are of length $p$ and $(p, q) \in\{(3,6),(4,4),(6,3)\}$, and proven an analogue of Theorem 1 in this setting.

Corollary of Theorem 1. In the case above, the cyclic permutations $\tilde{u}$ and $\tilde{w}$ and the conjugating word $c$ may be chosen in such a way that when the disk is depicted as a square tiling, the sides labelled by $c$ are straight lines (i.e., the word $c$ is composed entirely of letters of a single parity).

In the augmented Dehn presentation associated to an alternating elementary link projection, chains correspond in a natural way to certain sequences composed alternatingly of link crossings and of the regions formed in the plane by the link projection image. We call such sequences strolls. If two strolls of equal length bear a certain technical relationship to one another, we say that the two strolls run on parallel tracks.

Theorem 2. Suppose we have the augmented Dehn presentation associated to an alternating elementary link projection. Given a $2 \times n$ reduced rectangular diagram, consider it as a pair of chains with a common side of length $n$. Then either the two corresponding strolls run on parallel tracks, or else the label of the common side of 
the two chains has the form $\left(x^{\epsilon} y^{-\epsilon}\right)^{\frac{n}{2}}$ if $n$ is even and $\left(x^{\epsilon} y^{-\epsilon}\right)^{\frac{n-1}{2}} x^{\epsilon}$ if $n$ is odd (where $x$ and $y$ are distinct unsigned generators and $\epsilon \in\{1,-1\}$ ).

Corollary to Theorem 2. Suppose we have the augmented Dehn presentation associated to an alternating elementary link projection. A word which contains a subword $x^{\epsilon} y^{-\epsilon} z^{\epsilon}$ (where $x, y$, and $z$ are distinct unsigned generators and $\epsilon \in\{1,-1\}$ ) labels the side of no more than two distinct chains.

Suppose we have a $\mathrm{C}^{\prime \prime}(4)-\mathrm{T}(4)$ presentation with all relators of length four. If the first and last letters of a chain word are inverses (but the second letter is not the inverse of the next-to-last letter), then the corresponding chain is said to be circular. We can cyclically permute a circular chain by moving the first 2-cell to the end, thus producing another circular chain.

Theorem 3. Suppose we have the augmented Dehn presentation associated to an alternating elementary link projection. Then there are at most two (cyclically distinct) circular chains with a side labelled by a given word of the form $\left(x^{\epsilon} y^{-\epsilon}\right)^{n}$. In the case that there are two, the link projection image has a certain "helical" form (in some sense, the link is roughly a $(2, m)$-torus link).

Theorem 4. Suppose we have an alternating elementary link projection, and its associated augmented Dehn presentation. Suppose that the length of a cyclic geodesic word $w$ is $n$ and that the number of link crossings is $k$. If $u$ is a cyclic geodesic which is conjugate to $w$, then there is a conjugacy diagram for $w$ and a cyclic permutation of $u$ which is a subdiagram of a tiling requiring no more than $2 k n$ squares.

Once a maximal (non-repeating) tiling for a cyclic geodesic word $w$ has been produced (this tiling is unique), to search the tiling for cyclic permutations of a particular cyclic geodesic word $u$ requires quadratic time (see section 5). So the total computation time (given cyclic geodesics) is cubic; for arbitary words, it is bounded by a polynomial of degree no more than five.

Remark. One can examine the fundamental group of the complement of a spatial graph (graph embedding in $\mathbb{R}^{3}$ ) in the same way; the corresponding restrictions necessary for the augmented presentation to satisfy small cancellation conditions are looser. If the graph has all vertices of even degree, the group presentation has parity (again, you may checker the regions formed by the projection to the plane). So the same algorithm derived in section 5 for conjugacy applies. However, I have not attempted to verify whether the algorithm is polynomial-time; the methods of sections 6 and 7 may not generalize.

\section{Fundamentals}

2A. Some link terminology. Any tame link is ambient isotopic to a finite union of line segments, arranged in such a way that in the projection to the plane no node between line segments maps to a double point. We assume this type of projection (known as regular).

The projection image of a link may be modified slightly to indicate which strand is uppermost at each crossing, producing a picture called the link diagram. We will call the unmodified link projection image on the plane, together with the domains of the link projection image's complement in the plane, the link universe. We often 
treat the link universe as a 2-complex, letting the crossings become the vertices and so forth.

If at each vertex in the link universe four distinct regions meet, we say that the projection is untwisted; it is no loss of generality to assume that a regular link projection has this property (just use Reidemeister moves to remove twists).

If an alternating untwisted link projection has a connected image, then the link $L$ is not split: there is no homeomorphic image of a sphere $S^{2}$ in $\mathbb{R}^{3} \backslash L$ which separates pieces of the link $[\mathrm{M}]$.

Some untwisted link projections are said to be elementary, which means that two distinct regions of the link universe have at most one common edge in their boundaries.

Every (alternating) prime knot is ambient isotopic to some knot with an (alternating) elementary link projection $[\mathrm{Scb}],[\mathrm{LS}]$. If an alternating link projection is elementary, then the link is prime $[\mathrm{M}]$.

Each link in this paper is assumed to have a (fixed) alternating elementary projection. We are therefore dealing with all alternating prime links.

For simplicity of the arguments, we will assume that the link projection image is connected and has at least one crossing - i.e., the link is not split and is not the unknot. These assumptions are not necessary, however [J1].

2B. Dehn presentation. The Dehn presentation of the link group is given as follows. We are given a (fixed) regular link projection $p$ of the link $K$. Label the domains of $\mathbb{R}^{2} \backslash p(K)$ by $x_{0}, x_{1}, \ldots, x_{n}$, starting with the unbounded domain. These will be the names of the group generators. Fix a base point above the link. For each region $x_{i}$ there is a loop (loop-class representative) which descends from the base point, pierces the plane though region $x_{i}$, and returns upwards via the unbounded region $x_{0}$. Each crossing generates a relator as follows: at each crossing, four distinct regions meet (see Figure 1).

The set of relators arising from this crossing is the closure under the operations of cyclic permutation and inverse of the word $x_{a} x_{b}^{-1} x_{c} x_{d}^{-1}$. (This word merely says that a loop based above the link, piercing the plane downwards through region $x_{a}$ and returning upwards by piercing region $x_{b}$, can be slid through the crossingleaving the base point fixed - to become a loop going down through $x_{d}$ and returning via region $x_{c}$.) In addition to relators arising from the crossings, there is an additional relator $x_{0}$. The significance of this additional relator is that a loop not piercing any bounded region is homotopically trivial. With the exception of $x_{0}$, all of the generators are of infinite order (in the case of an elementary alternating projection).

The presentation we will use is obtained by deleting the odd relator $x_{0}$. The new presentation - call it the augmented (Dehn) presentation - presents the free product of the link group and the infinite cyclic group. (To see this, add a circle to the link so that its projection image is disjoint from, and completely encircles, that

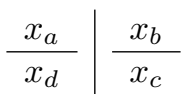

Figure 1 
of the link. The Dehn presentation of the new link is equivalent to the augmented presentation of the old link.)

Remark. It may not be obvious why we would choose to work with the augmented group instead of directly with the link group. First let us note that the augmented group distinguishes links precisely as well as the link group itself: this is a consequence of a theorem of Papakyriakopoulos $[\mathrm{P}]$, that the group of a link is a proper free product if and only if the link is split (in which case it is the free product of the link groups of the split components).

Secondly, solving the Word and Conjugacy Problems in the augmented group solves them in the link group. The automorphism $x_{0} \mapsto x_{0}, x_{i} \mapsto x_{i} x_{0}^{-1}($ for $i \neq 0$ ) takes the augmented presentation to the obvious free product form.

Finally, the augmented presentation has some very nice combinatorial properties that the Dehn and Wirtinger presentations do not. We shall make heavy use of certain small cancellation properties, and also of the curious alternating nature of the relators (both in parity and in sign). To explain these properties fully, it will be expedient to have some conventions about small cancellation diagrams.

2C. Digression: a word about diagrams. For conventions about graphs and small cancellation diagrams see [LS], [GeS1]. It should be noted that all our diagrams are reduced (so the degree of any interior vertex is always greater than two), and that all our diagrams are simply connected. Recall that a diagram having at least one 2-cell, and having no cut vertices, is called a disk. A diagram is made up of a union of disks (called the disk components of the diagram) and trees.

One minor change of terminology is that an edge path of length one (sometimes called a directed edge or oriented edge) will be called an edge traversal $T:[0,1] \rightarrow \bar{e}$ (where $e$ is a 1-cell and $T(0)$ and $T(1)$ are its endpoints). The inverse traversal is $T^{-1}:[0,1] \rightarrow \bar{e}$ by $T^{-1}(r)=T(1-r)$. Every edge is associated with a (fixed) inverse pair of edge traversals. When a graph is depicted pictorially as a set of points and line segments, a traversal of an edge is indicated by drawing an arrowhead on the edge in the direction desired.

We represent a labelling of a graph (from a group presentation alphabet) pictorially by drawing on each edge one of two possible arrowheads, and writing next to it the label of the corresponding edge traversal. This is unambiguous, since the label of the inverse edge traversal is the inverse of this letter.

We shall require (with no loss of generality) that the boundary cycle label of any disk component of a diagram be cyclically freely reduced (i.e., there are no consecutive edge traversals with labels $x^{\epsilon}$ and $x^{-\epsilon}$ ).

\section{Dehn PRESEntation Properties}

The augmented Dehn presentation of an elementary alternating link has three peculiarities that make it particularly amenable to geometric methods: it satisfies small cancellation conditions $\mathrm{C}^{\prime \prime}(4)$ and $\mathrm{T}(4)$ (with all relators of length four), the generators have a natural parity, and the presentation is alternating.

Let a two-letter subword of some relator be called a pair. Each of the three properties may be expressed in terms of pairs.

3A. Small cancellation conditions. Given a set of words which is closed with respect to inverse and cyclic permutation, we say that a nonempty word $b$ is a piece 
(relative to the set of words) if we have distinct words $r_{1}$ and $r_{2}$ in the set such that $r_{1} \equiv b c_{1}$ and $r_{2} \equiv b c_{2}$.

For an alternating elementary link projection, the augmented presentation satisfies the small cancellation hypotheses below:

Condition $\mathbf{C}^{\prime \prime}(\mathbf{4})$. No defining relator is a product of fewer than four pieces. (If all the relators are four letters long, this says that no pair is a piece, i.e., knowing two successive letters determines a relator uniquely up to cyclic permutation.)

Condition $\mathbf{T}(4)$. Suppose that $r_{1}, r_{2}$, and $r_{3}$ are any three defining relators such that no two of the words are inverses of each other. Then at least one of the three products $r_{1} r_{2}, r_{2} r_{3}$, and $r_{3} r_{1}$ is freely reduced without cancellation. (I.e., if ab and $b^{-1} c$ are pairs (where $c \not \equiv a^{-1}$ ), then ac is not a pair.)

In a diagram for a presentation satisfying $\mathrm{C}^{\prime \prime}(4)$ (and such that each relator is of length four), an edge path which is common to the boundaries of two distinct regions (and is therefore labelled by a piece) has length no more than one. A (reduced) diagram for a presentation satisfying $\mathrm{T}(4)$ has the property that the degree of any interior vertex is at least four.

It is easy to see that the $\mathrm{T}(4)$ condition is met for the augmented presentation associated to any regular link projection, since the letters of a pair are opposite in parity (see the definition of the augmented presentation). If the link is alternating and elementary, $\mathrm{C}^{\prime \prime}(4)$ holds also: Note that $x_{a}^{\epsilon} x_{b}^{-\epsilon}$ is a pair if and only if regions $x_{a}$ and $x_{b}$ of the link universe are neighbors. Neighboring regions have only one common edge, since the projection is elementary. Since the projection is alternating, the pair for $\epsilon=-1$ arises from the crossing at one endpoint of the edge between $x_{a}$ and $x_{b}$, and the pair for $\epsilon=+1$ arises from the crossing at the other endpoint of this edge.

So for alternating elementary links, the augmented presentation satisfies $\mathrm{C}^{\prime \prime}(4)$ and $\mathrm{T}(4)$. But since all the relators have length exactly four, these conditions become more powerful.

Consider a square with each of the four sides labelled by a letter, in such a way that reading clockwise around the boundary gives a relator. (Such a square, of course, is just a diagram which consists of a single 2-cell and its labelled boundary. For this reason we call such a diagram a relator square.) Rotating the square $90^{\circ}$ corresponds to a cyclic permutation of the relator; flipping the square corresponds to taking an inverse. If we wish to use squares to tile a surface of nonpositive curvature, at least four squares must meet at any vertex. If we are tiling with relator squares and we have a right angle with labelled edge traversals, at most one relator square exists which can be placed to fill this angle.

This idea of relators as squares motivates much of what we will be doing.

In an arbitrary finite group presentation, the set of lengths of all words representing a group element has a least element. A word achieving this minimal length for the group element it represents is called a geodesic. It is easy to see that in a $\mathrm{C}^{\prime \prime}(4)-\mathrm{T}(4)$ group with all relators of length four, a geodesic must be freely reduced (i.e., have no subword $x^{\epsilon} x^{-\epsilon}$ ) and have no subword which is three-fourths of some relator. However, these conditions do not suffice to identify the geodesics. For this, we will need another definition.

Definition: Chain. A chain (or strip) is a reduced disk having the form shown in Figure 2, where $n \geq 1$. 


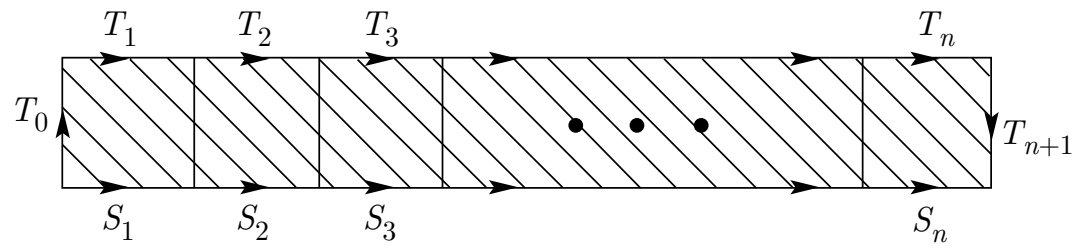

FIGURE 2

We say that a disk has a chain along the boundary if there is a subdiagram like Figure 2 such that the indicated edge path $\alpha=T_{0} T_{1} T_{2} \ldots T_{n} T_{n+1}$ is a subpath in a boundary cycle of the disk; in this case, the subpath $T_{1} T_{2} \ldots T_{n}$ is called the outer link path of the chain (relative to the disk). (Note that a chain along the boundary of a disk is thought of as a chain subdiagram as described, together with a specified outer link path.) We say that a word is a chain word if it forms the edge path label of the path $\alpha$ for some chain as above.

Remark. In [GeS1], chain word is used to mean what is herein known as outer link path label.

In this paper we will only deal with chains when considering a finite presentation satisfying $\mathrm{C}^{\prime \prime}(4)-\mathrm{T}(4)$ and with all relators of length four.

At first glance, it may seem that it would be difficult to determine whether a word were a chain word, but in fact it is linear-time for a finite $\mathrm{C}^{\prime \prime}(4)$ presentation: It is constant-time to check whether a particular two-letter word is a pair, and a pair uniquely determines a relator $\left(\mathrm{C}^{\prime \prime}(4)\right)$. So if a word which consists of all but the last letter of some chain word is called a chain prefix, and a chain suffix is defined analogously, we note that in a $\mathrm{C}^{\prime \prime}(4)$ presentation, a chain prefix (or suffix) uniquely determines its chain diagram, and hence in particular the missing letter of the chain word.

Note that three successive letters of a relator form a chain word, with $n=1$. It is easy to see that chain words are not geodesic: let the inner link path of the chain above be $\beta=S_{1} S_{2} \ldots S_{n}$; the label of $\beta$ equals the label of $\alpha$ and is shorter.

A consequence of Lyndon's Curvature Theorem is that a $C^{\prime \prime}(4)-T(4)$ disk has at least four chains on its boundary whose outer link paths have disjoint edge sets [GeS1]. (For example, consider a disk consisting of a single relator square. This disk actually has four chains on its boundary, in the sense that each of the four sides takes its turn being the outer link path of a chain of length one.)

Suppose a group presentation satisfies $\mathrm{C}^{\prime \prime}(4)-\mathrm{T}(4)$ and all relators are of length four. If a disk has exactly four such chains on its boundary, then it may be represented as a square tiling (with simple boundary curve) in the Euclidean plane; moreover, in the cyclic sequence of boundary vertices of the disk, between any two boundary vertices of degree four is a boundary vertex of degree two [J2]. A consequence is:

Geodesic Characterization Theorem [AS2]. A word in a $C^{\prime \prime}(4)-T(4)$ presentation is geodesic if and only if it is freely reduced and contains no chain subwords.

Remark. This folklore theorem is not stated formally in [AS2], although it follows from a lemma therein. It is clearly understood in [GeS1], but neither stated nor proved. There are formal proofs in [J1], [K], [J2]. 
Consequently in finite $\mathrm{C}^{\prime \prime}(4)-\mathrm{T}(4)$ group presentations, it is no loss of generality to assume that a word is geodesic, because the above gives a (quadratic-time) algorithm for replacing an arbitrary word by a geodesic representative of the same group element (delete occurences of $x^{\epsilon} x^{-\epsilon}$ and replace chain words by the labels of the inner link paths of their chain diagrams).

Geodesic Completion Theorem [J2]. Suppose a presentation satisfies $C^{\prime \prime}(4)$ and T(4), and has all relators of length four. Then a geodesic word uniquely determines a square tiling (which is a diagram) bounded by (but not necessarily filling) a rectangle in the Euclidean plane, having the properties:

1. the word labels a geodesic rectilinear edge path from one corner of the rectangle to the opposite corner; and

2. the diagram has the smallest number of relator squares to satisfy the requirement that if a word amounting to at least half of a relator labels an edge path in the diagram, then there is a relator cell in the diagram whose boundary includes that edge path.

This diagram encodes all geodesic words equal to the given word as the set of all labels of geodesic rectilinear edge paths which are path-homotopic to the edge path labelled by the original geodesic. The diagram also embeds in the Cayley 2-complex of the group presentation, and has the minimal number of 2-cells for all diagrams with that boundary cycle label.

Since we will later want to produce such tilings, it is worthwhile to include the construction of this tiling. We need some more nomenclature:

If there is a letter $b$ such that $a b$ and $b^{-1} c$ are both pairs (where $c \not \equiv a^{-1}$ ), then we call $a c$ a sister-set. In a T(4) presentation, no sister-set is a pair. (A consequence for $\mathrm{C}^{\prime \prime}(4)-\mathrm{T}(4)$ presentations is that (inner or outer) link paths of a chain are labelled by geodesic words - this follows from the Geodesic Characterization Theorem.)

Suppose a group presentation satisfies $\mathrm{C}^{\prime \prime}(4)-\mathrm{T}(4)$ and all relators have length four. Imagine a disk of three relator cells in the shape of an "L." The edge path of length two on the inner crook of the " $\mathrm{L}$ " might be a pair (that is, there may be a fourth relator cell that may be placed so as to fill the right angle), but it may not. If a sequence of two letters $a b$ labels some such edge path but is not a pair, we call it a pseudo-pair.

Remark. Given a $\mathrm{C}^{\prime \prime}(4)-\mathrm{T}(4)$ group presentation with all relators of length four, it is easy to identify the pairs, sister-sets, and pseudo-pairs, using matrix powers of the incidence matrix of the Whitehead graph.

In a group presentation with parity, no pseudo-pair is a sister-set (because this implies the existence of a disk with interior vertex of degree five). This need not be true in general. Also, the two letters of a pair or pseudo-pair are always opposite in parity, whereas those of a sister-set always have the same parity.

Now we can construct the tiling of the last theorem.

Consider the Cartesian plane as a potential tiling by letting points with both coordinates integers be potential vertices; open line segments of the form $((n, m)$, $(n+1, m))$ or the form $((n, m),(n, m+1))$ (where $n, m \in \mathbb{Z})$ be potential edges; and unit squares with corners at potential vertices be potential 2-cells. When we want to include one of these in a certain tiling, we say that we "choose" it for the tiling. 
Definition. We are given a geodesic word in a presentation satisfying $\mathrm{C}^{\prime \prime}(4)$ and $\mathrm{T}(4)$ and having all relators of length four. We choose the point $(0,0)$ as a vertex. If the word is empty, we are done. Otherwise, starting at $(0,0)$, choose the vertex a displacement $\hat{\imath}$ away and also choose the edge between them. Let the edge traversal along the new edge to the new vertex be labelled by the first letter of the word. If the word has more letters remaining, then for each in succession start at the last vertex chosen and choose a vertex and corresponding edge either $\hat{\imath}$ or $\hat{\jmath}$ away, according to the following criterion: If the immediately preceding letter forms a pair (or a pseudo-pair which is not a sister-set) with the new letter, change direction from that taken most recently; otherwise keep going the same way. Label the edge traversal from the old vertex to the new vertex by the corresponding letter of the word. Continue with the next letter of the word.

Suppose that the final vertex chosen in the above process is at the point $(n, m)$.

The graph resulting from the above process is called the standard embedding of the geodesic word.

Represent the standard embedding by $C_{0}$. Consider the following iterative process: for each pair of consecutive edges in the boundary of $C_{i}$ which form a right angle in the plane, but which are not contained in the boundary of some common relator square, look at the label $x y$; if $x y$ is a pair, choose the unit square determined by these edges, and also choose this square's remaining two boundary edges. Label the traversals of the new edges as indicated by the relator determined by $x y$. The new diagram is $C_{i+1}$. Continue this process until the resulting diagram has the property that if an edge path is labelled by a pair, then the edge path is included in the boundary of some 2-cell in the diagram. The result is the square tiling desired, known as the geodesic completion of the word.

Note that this is a finite process, since the maximal possible tiling fills the $n \times m$ rectangle.

The form in which the theorem above will be used in this paper is this: A (maximal) simply connected tiling of relator squares in the Euclidean plane is uniquely determined within a rectangular boundary by specifying the (geodesic) label of a geodesic edge path from one corner of the rectangle to the opposite corner. Also (because the diagram embeds in the Cayley 2-complex and is minimal, i.e., has the smallest number of 2-cells for its boundary cycle label) in any disk which is a square tiling, edge paths are geodesic if and only if their labels are geodesic words.

In a diagram for a group presentation with all relators of length four, every 2-cell has exactly four sides, so it is natural to treat them as squares geometrically, i.e., give each corner an angle of $\frac{\pi}{2}$. We can then define an angle at a boundary vertex of a disk which measures how much the boundary deviates from a straight line at that vertex. (This definition is taken from [GeS1].)

Suppose a group presentation satisfies $\mathrm{C}^{\prime \prime}(4)$ and $\mathrm{T}(4)$ and has all relators of length four. For a vertex $v$ of a disk, let $\sigma_{v}$ denote the sum of the angles in the disk incident on $v$. That is, $\sigma_{v}=\frac{\pi}{2} \times$ the number of relator squares incident on $v$. For $v$ in the boundary of a disk, define the turning angle at $v$ (relative to that disk) to be $\tau(v)=\pi-\sigma_{v}$. Since there must be at least one incident relator square, $\tau(v)$ can be no more than $\frac{\pi}{2}$, and all other possible values are nonpositive. That is, $\tau \in\left\{+\frac{\pi}{2}, 0,-\frac{\pi}{2},-\pi, \ldots\right\}$.

Recall that in such a group presentation, a disk which has exactly four chains on its boundary may be represented as a tiling of squares in the Euclidean plane. 
In such a tiling, exactly four squares are incident on each interior vertex, and one, two, or three squares are incident on any boundary vertex. To say it another way, there are only three kinds of turning angles: $+\frac{\pi}{2}, 0$, and $-\frac{\pi}{2}$. Moreover, along any segment of the boundary between two turning angles of $-\frac{\pi}{2}$ there is a turning angle of $+\frac{\pi}{2}[\mathrm{~J} 2]$.

Consider an edge path of length two in the boundary of an arbitrary disk in a presentation as above. If the middle vertex of such an edge path has a turning angle of $+\frac{\pi}{2}$, then the edge path is labelled by a pair; if the turning angle is 0 , then the label is a sister-set; if the turning angle is $-\frac{\pi}{2}$, then the label may be either a pair or a pseudo-pair.

3B. Parity. If a presentation has all relators of even length, and the property that each generator pair $x_{i}$ and $x_{i}^{-1}$ can be assigned a parity in such a way that the letters of each relator alternate in parity, we say that the presentation has parity. At any interior vertex of a diagram for such a presentation, labels of successive edge traversals alternate in parity. Therefore the degree of any interior vertex is always even.

Consider a regular link projection. The projection image on the plane divides the plane into a 2-colorable map. The group generators $x_{i}$ and $x_{i}^{-1}$ of the associated augmented presentation inherit the parity of the link universe region $x_{i}$. Note that the letters of any relator alternate in parity. Therefore parallel sides of a relator square have edge traversals of the same parity, and perpendicular sides have opposite parity. Since a (reduced) diagram cannot have a vertex of interior degree two, this implies that the augmented group satisfies $\mathrm{T}(4)$.

Suppose in a group presentation with parity we have a disk which happens to be a tiling of squares in the Euclidean plane. Then traversals of parallel edges have labels of the same parity, and traversals of perpendicular edges have labels of opposite parity. In particular, this is true of a chain. So the first and final letter of a chain word are of one parity, and the remaining letters are all of the other parity.

In a $\mathrm{C}^{\prime \prime}(4)-\mathrm{T}(4)$ presentation with parity and all relators of length four, the standard embedding of a geodesic word (defined in preceding section) may be alternatively defined by going straight if the new letter has the same parity as the last, and turning if they are of opposite parities.

Corollary. If a $C^{\prime \prime}(4)-T(4)$ presentation has parity and all relators of length four, then the geodesic completion of a geodesic word having $n$ letters of one parity and $m$ of the other is contained within an $n \times m$ rectangle.

3C. Alternating sign. Suppose we have a group presentation in which all the relators are even in length and alternating in sign, and that no proper subword of a relator represents the identity element. We say such a presentation is alternating.

Remark. Such a presentation defines a free product with the infinite cyclic group. Given any finite group presentation, we can write a presentation of this form for the corresponding free product: add a new letter $y$ and its inverse to the alphabet, and use the embedding $x_{i} \mapsto x_{i} y^{-1}$ for all $x_{i}$.

Suppose we have an alternating presentation. Consider a vertex $v$ on the boundary of a disk. Consider the set of all the edges of the disk incident on $v$; for each, there is a traversal that starts at $v$ and ends distance one away. Exactly two of the incident edges are boundary edges of the disk, so consider the labels of successive traversals in a sequence from one of the boundary edges to the other. 
Because the relators are alternating in sign, this sequence of labels is composed entirely of letters of a single sign.

Now consider the analogous cyclic sequence of letters for an interior vertex of a diagram. If we ignore for a moment one of the incident regions, the vertex is a boundary vertex of a disk. Therefore this sequence, too, is composed entirely of letters of a single sign.

It follows that in a disk from an alternating presentation, the letters in the label of any edge path alternate in sign.

The major consequence for this paper is given as the first proposition in the following section.

\section{Conjugacy diagrams}

We say that a word is a cyclic geodesic if all cyclic permutations of the word are geodesic words.

Suppose we have a $\mathrm{C}^{\prime \prime}(4)-\mathrm{T}(4)$ presentation. Note that, given a non-geodesic word, we can replace it by a word (representing the same group element) which is geodesic by an iterative process of removing cancelling pairs and replacing chain subwords by their inner link path labels. Given a geodesic word, we can replace it by a conjugate word that is a cyclic geodesic: Cyclically permute the geodesic word by one letter; this is a conjugation of the word, and while the word thus produced may not itself be geodesic, it can be replaced by a equal word which is geodesic. Cyclically permute and repeat. Eventually this length-decreasing process produces a word which is conjugate to the original word and is a cyclic geodesic. This requires no worse than quadratic time, by a tiling argument (cyclically permute the geodesic completion).

If a cyclic permutation of a word is equal to a cyclic permutation of a second word, we say that the two words are cyclically equal.

In this paper, a conjugacy diagram for words $u$ and $w$ is a (simply connected) reduced diagram with boundary cycle label of the form $c^{-1} w c u^{-1}$, where $c$ is some word.

For an arbitrary presentation, there is an easy, pictorial proof that if two cyclic geodesic words are conjugate via a conjugacy diagram with a cut vertex, then either the two words are cyclically equal, or there is a conjugating word (having shorter length than the conjugating word in the original diagram) between a cyclic permutation of the one word and a cyclic permutation of the other so that the conjugacy diagram is a disk (see [LS] or [J1]).

In a $\mathrm{C}^{\prime \prime}(4)-\mathrm{T}(4)$ presentation, the time necessary to determine whether two cyclic geodesics are cyclically equal is certainly no worse than quartic. (Check whether the first word is equal to any of the cyclic permutations of the second - each check takes quadratic time - then repeat process for remaining cyclic permutations of the first word.) In fact, we'll see in section 5 that the time required is only quadratic. So for now we'll consider conjugacy diagrams for cyclic geodesic words $u$ and $w$ which are not cyclically equal, and for which the choice of conjugating word $c$ is minimal. This implies that neither $u$ nor $w$ is trivial (else they both are, a contradiction) and that $c$ is not trivial.

Proposition 4.1. Suppose that a group presentation is alternating. If $u$ and $w$ are conjugate cyclic geodesic words which are not cyclically equivalent, then $u$ and $w$ are alternating words of even (non-zero) length. 
Proof. There are cyclic permutations $\tilde{w}$ and $\tilde{u}$ of $w$ and $u$ such that a conjugacy diagram with boundary cycle label $c^{-1} \tilde{u} c \tilde{w}^{-1}$ has no cut vertices. Therefore not only must $\tilde{w}, \tilde{u}$, and $c$ all be of alternating sign, but also $c$ must start with a letter of the same sign as the first letter of $\tilde{u}$ and of opposite sign to the last letter of $\tilde{u}$, and end with a letter opposite in sign to the first letter of $\tilde{w}$ and of the same sign as the last letter of $\tilde{w}$.

Henceforth for simplicity we shall refer to the words labelling the conjugate sides of the disk simply as $u$ and $w$. We hope, by analyzing the geometry of the disk, to find out more about $u, w$, and the conjugating word $c$ in the case of the augmented Dehn presentation associated to an alternating elementary link diagram. We will first show that the disk has exactly four chains on its boundary, and thus can be treated as a square tiling.

We think of the disk as a rectangle; there are four "boundary edge paths" $\alpha, \beta$, $\gamma_{o}$, and $\gamma_{t}$, with labels $u, w, c$, and $c$, respectively (where $\gamma_{o}$ goes from the origin of $\alpha$ to the origin of $\beta$, and $\gamma_{t}$ connects the terminus of $\alpha$ to the terminus of $\beta$ ). Let a boundary vertex of the disk which lies on two of these four boundary edge paths be called a "corner" of the disk.

Henceforth we will be assuming that the presentation satisfies $\mathrm{C}^{\prime \prime}(4)-\mathrm{T}(4)$ and that all relators are of length four. So keep in mind that there are at least four chains on the boundary of the disk [GeS1]. Since each of the words $w, u$, and $c$ is geodesic, none has a chain subword. So any chain on the boundary of the disk must include at least one edge of $\alpha \cup \beta$, and at least one edge of $\gamma_{o} \cup \gamma_{t}$. To put it another way, at least one corner vertex of the disk must be included on each chain. A chain can touch two of the corner vertices only if its outer link path includes the entirety of one of the four boundary edge paths. (If the presentation has parity, then the label of that edge path is composed entirely of letters of a single parity.) Also, if two chains have letters on a single edge of the boundary, this edge must necessarily have an endpoint at a corner of the diagram, and that corner must have a turning angle of $+\frac{\pi}{2}$-in the sequence of non-zero turning angles on the boundary of the disk, this is the middle of a sequence of three consecutive $+\frac{\pi}{2}$ angles. Such a corner will be called a magic corner. If three chains have letters on a single one of the edge paths $\alpha, \beta, \gamma_{o}$, or $\gamma_{t}$, then at least one of the corners of that edge path must be magic.

Recall that a cyclic geodesic word is one for which any cyclic permutation is a geodesic word. Clearly an ordinary geodesic must be cyclically freely reduced to be a cyclic geodesic, but we must also ensure that no suffix of the word, when concatenated with a disjoint prefix, forms a chain. There is a relatively straightforward means of checking this. If a word $w$ begins with a chain suffix, we denote this subword by $f(w)$. If $w$ ends with a chain prefix, we call that subword $l(w)$.

If the last letter of $w$ together with the first is a sister-set, then the word fails to be a cyclic geodesic only if $f(w)$ and $l(w)$ are both defined (and non-overlapping) and $l(w) \cdot f(w)$ is a chain.

If the last letter $w_{n}$ of $w$ together with the first letter $w_{1}$ is a pair, then the word fails to be a cyclic geodesic only if either $l(w) \cdot w_{1}$ is a chain, or if $w_{n} \cdot f(w)$ is a chain (where the two parts are disjoint in $w$ ).

Remark. If the presentation has parity, there is a "quick and dirty" way to simplify the above, because the letters labelling the outer link path of a chain are all of a single parity, and the first and last letter of the chain word are of the opposite 
parity. So instead of actually looking for a chain suffix at the beginning of $w$, just take all the letters up to and including the first letter representing a change of parity; and analogously for $l(w)$.

The first and the last letter of a chain word are called the clasps; the rest are called the link letters. We also use the terms clasps and (outer) links for the edges labelled by these letters if the chain word labels an edge path in the boundary of a disk.

What we want to do now is analyze the ways in which two distinct chains in the boundary of the conjugacy disk can be situated relative to the same edge path $\delta \in\{\alpha, \beta\}$. We will show that certain configurations are impossible, either because the label $v$ of $\delta$ is a cyclic geodesic, or because of parity considerations.

If a chain has a clasp on $\delta \in\{\alpha, \beta\}$ but no (outer) link letters, then we say the chain is in position "Up" relative to $\delta$. If all of the link letters (and at most one clasp) are on $\delta$, the chain is in position "Down." If some of the link letters are on $\delta$ and some not, then the position is said to be "Partial."

The "Down-Down" Lemma 4.2. If $u$ and $w$ are cyclic geodesics in a $C^{\prime \prime}(4)$ $T(4)$ presentation with all relators of length four, and $u$ and $w$ are not cyclically equal, then it is not possible for two distinct chains to be in position "Down" relative to $\delta \in\{\alpha, \beta\}$, unless the final link letter and clasp of one chain are the initial clasp and first link letter of the other.

If the presentation has parity, it is impossible for the chains to overlap in such a pair. It follows that there are at least two chains with links on the union of $\gamma_{o}$ and $\gamma_{t}$.

Proof. Suppose there are two chains in position "Down" relative to $\delta$. Then the word $v$ labelling $\delta$ starts with a chain suffix and ends with a chain prefix, so $f(v)$ and $l(v)$ are both defined. Since we can't have two consecutive turning angles of $\frac{\pi}{2}$ along $\delta$, we note that the final letter (clasp) of the prefix subword $f(v)$ is disjoint from the first letter (clasp) of the suffix subword $l(v)$.

Since at either end $\delta$ (labelled by $v=v_{1} v_{2} \ldots v_{n}$ ) meets an edge path $\gamma_{i}$ labelled by the conjugating word (let the conjugating word start with letter $a$ ) at a turning angle of $\frac{\pi}{2}$, it follows that $a^{-1} v_{1}$ and $v_{n} a$ are pairs. So either $v_{n} v_{1}$ is a sister-set, or else $v_{n} v_{1}$ is a cancelling pair (but this is impossible, since $v$ is cyclically freely reduced). So unless $f(v)$ and $l(v)$ overlap in a pair, $l(v) \cdot f(v)$ is a chain word, which implies that $v$ is not cyclically geodesic - a contradiction.

Now suppose that the presentation has parity. Then the last letter of $f(v)$ has the same parity as $a$ (which is the first letter of the conjugating word), and so does the first letter of $l(v)$. If the last letter of $f(v)$ is also the first link letter of $l(v)$, then the pair which constitutes the intersection of the two subwords has both letters of the same parity, which is impossible.

There are at least four chains on the boundary of the conjugacy disk, and each must have at least one letter either on $\gamma_{o}$ or on $\gamma_{t}$. We have just established that in the case of parity, at most two chains have only a clasp on $\gamma_{o} \cup \gamma_{t}$.

We will henceforth assume parity.

If a chain on the boundary of the disk includes all of $\delta$ in its outer link path, then clearly at most three chains touch $\delta$. The above lemma implies that when there is not such a chain along all of $\delta$, then the two corner vertices at either end of $\delta$ can't both be magic corners; therefore, again, at most three chains have letters on $\delta$. So 
there are at most six chains on the boundary of the disk. We'll show that there are exactly four.

Let us begin by making a small observation about the conjugating word $c$. Suppose that $c$ has no pairs, but does not label the link path of any chain. Then any chain with letters on $\gamma \in\left\{\gamma_{o}, \gamma_{t}\right\}$ must have a single clasp on $\gamma$, no other letters. This contradicts the above lemma, since there are at least four chains total, each touching at least one of $\gamma_{o}$ and $\gamma_{t}$. So henceforth we may assume that $c$ either contains at least one pair, or else labels the link path of some chain-in particular, is entirely of a single parity.

We first consider the possibility that the conjugating word $c$ contains no pairs. This implies that if a chain has an edge on $\gamma \in\left\{\gamma_{o}, \gamma_{t}\right\}$, then either $\gamma$ contains only a clasp of the chain, or else all of $\gamma$ lies along the outer link path of the chain. Since at least two chains have links on the union of $\gamma_{o}$ and $\gamma_{t}$, it must be that all of $\gamma_{o}$ lies along the link path of a chain, and all of $\gamma_{t}$ lies along the link path of a chain. Both of these chains have edges on both $\alpha$ and $\beta$. There must be at least two other chains on the boundary of the disk; at most three may have letters on either of $\alpha$ or $\beta$, and we have already accounted for two chains touching each. So there is one chain with links on $\alpha$ and one with links on $\beta$ (both chains necessarily in the "Down" position). (Note that therefore, each of $\alpha, \beta$ has at least one magic corner.) This makes the maximum number of chains four.

Now we may suppose that the conjugating word $c$ contains at least one pair. We will prove several lemmas before asserting that the number of chains is exactly four.

The "Up-Up" Lemma 4.3. If $u$ and $w$ are cyclically reduced words in a $C^{\prime \prime}(4)$ $T(4)$ presentation with all relators of length four, if $u$ and $w$ are not cyclically equal, and if the conjugating word $c$ contains at least one pair, then it is not possible for two distinct chains to be in position "Up" relative to $\delta \in\{\alpha, \beta\}$.

It follows that there must be at least two chains with links on $\alpha \cup \beta$.

Proof. Suppose such a diagram did exist. Since the two conjugating sides are labelled by the same word $d \in\left\{c, c^{-1}\right\}$ which by assumption contains at least one pair, the link letters and terminal clasps of the two "Up" chains must be labelled by the same word, namely $f(d)$. Since $f(d)$ is a chain suffix, the first letter of this chain word is uniquely determined. This letter is the inverse of the first letter of $v \in\{u, w\}$, and it is also the last letter of $v$. This contradicts the assumption that $v$ is cyclically freely reduced.

The "Up-Partial" Lemma 4.4. If $u$ and $w$ are cyclic geodesics in a $C^{\prime \prime}(4)-T(4)$ presentation with parity and all relators of length four, and if the conjugating word c contains at least one pair, then it is not possible for two chains to be in positions "Up" and "Partial" relative to $\delta \in\{\alpha, \beta\}$.

Proof. Suppose such chains exist. Since $c$ contains at least one pair, each of the two chains has a chain suffix in the conjugating word $d \in\left\{c, c^{-1}\right\}$. As in the last proof, a chain suffix of either chain must be labelled by the chain suffix $f(d)$. Since a chain suffix of length $n$ uniquely determines a chain diagram of $n-12$-cells, the "Partial" chain actually concludes with a copy of the "Up" chain. So the "Partial" chain may be broken into two subchains at the point where it passes from $\delta$ to $\gamma \in\left\{\gamma_{i}^{ \pm}\right\}$, with the latter piece being a copy of the "Up" chain. For ease of notation, say that $\gamma=\gamma_{t}$ (so the label of $\delta$ is $w=w_{1} \ldots w_{n}$ ). Since the second part of the "Partial" 
chain is a copy of the "Up" chain, when the "Partial" chain is broken into two pieces the resulting chain word on the second piece is $w_{1}^{-1} \cdot f(c)$ (same as the chain word on the "Up" chain). Therefore the chain word on the first piece is $l(w) \cdot w_{1}$. If we can show that the subword $l(w)$ of $w$ is disjoint from the first letter of $w$ we are done, since then the cyclic permutation $w_{1}^{-1} \cdot w \cdot w_{1}$ of $w$ contains a chain subword $l(w) \cdot w_{1}$. This would be a contradiction, since $w$ is a cyclic geodesic.

Each of the "Up" and the "Partial" chain have a clasp on $\alpha$. Note that the clasp of the chain in the "Up" position cannot also be the clasp of the "Partial" chain, else the 2-cells of the two chains coincide; which is impossible, since the chains are of different lengths (the "Partial" chain contains link letters in $w$ as well as in $c$ ). If the clasp of the chain in the "Up" position is also the first link of the "Partial" chain, then its label is both a link letter of the "Partial" chain and the label of a perpendicular edge traversal (the interior edge at the other end of the edge path $\alpha)$. This is impossible by parity. So the clasp of the "Up" chain must be disjoint from the edges of the "Partial" chain - the "Partial" chain does not contain all of the edge path $\alpha$.

Therefore the cyclic permutation $w_{1}^{-1} \cdot w \cdot w_{1}$ of $w$ contains a chain subword $l(w) \cdot w_{1}$. This contradicts the assumption that $w$ is a cyclic geodesic.

Conjugacy Tiling Theorem 4.5. If $u$ and $w$ are conjugate (cyclically unequal) cyclic geodesics in a $C^{\prime \prime}(4)-T(4)$ presentation with parity and all relators of length four, then any conjugacy diagram which is a disk has exactly four chains on the boundary. Exactly two of the chain words include letters of $u$, unless the diagram is a rectangular array of squares (in which case each of $u, w$, and the conjugating word $c$ is composed of letters all of one parity).

Consequently the diagram is actually a tiling of a finite-area subset of the Euclidean plane by squares of side length one, with a non-self-intersecting boundary, and along any segment of the boundary cycle between two turning angles of $-\frac{\pi}{2}$ is a turning angle of $+\frac{\pi}{2}$.

Proof. We have already seen that there are no more than six chains on the boundary of the conjugacy disk, and there must be at least four; we want to show that there are precisely four. The tiling statement then follows (see section 3A).

We have already considered the case that the conjugating word $c$ contains no pairs. So suppose that $c$ does contain at least one pair. To have three chains with letters along the edge path $\delta$, one of the ends of $\delta$ must be a magic corner, and so there must be chains both of type "Up" and type "Down" there. So if there is a (distinct) chain touching the other end of $\delta$, it cannot have type "Down" (by the "Down-Down" lemma), type "Up" (by the "Up-Up" lemma), or type "Partial" (by the "Up-Partial" lemma). But this exhausts the possibilities. So there are at most two chains with letters along the edge path $\delta$. (Also, if only one chain touches $\alpha$, then three must touch $\beta$, a contradiction.) It follows that there are at most four chains on the boundary of the disk.

The "Partial-Partial" Lemma 4.6. Suppose $u$ and $w$ are cyclic geodesics in a $C^{\prime \prime}(4)-T(4)$ presentation with parity and all relators of length four, and suppose that $u$ and $w$ are conjugate but not cyclically equal. Then it is not possible for two chains of type "Partial" to have outer link letters along $\delta \in\{\alpha, \beta\}$.

Proof. Suppose such a diagram exists. We have already done the case that $c$ contains no pairs, so suppose that $c$ contains at least one pair. Also note that if a single 
chain has links including edges of $\gamma_{o}, \alpha$, and $\gamma_{t}$, then three chains must touch $\beta$, contradicting the last theorem. So both chains have the same chain suffix, namely $f(d)$ (where $d \in\left\{c^{ \pm}\right\}$), and so end with identical subchains. So we can break either chain into two subchains, where the chain word on the second subchain (in either case) is $a \cdot f(d)$ for some letter $a$. The first subchain has chain word $a \cdot f(v)$ in one case, and $l(v) \cdot a^{-1}$ in the other case (where $v$ is the label of $\delta$ ). The subwords $f(v)$ and $l(v)$ of $v$ cannot overlap by a single letter, else the 2-cells of the chains coincide (causing contradictions); they cannot overlap in a pair, because of parity. So $f(v)$ and $l(v)$ are disjoint subwords of $v$.

Say that $v=v_{1} \ldots v_{n}$. Note that $a v_{1}$ is a pair and $v_{n} a^{-1}$ is a pair. So $v_{n} v_{1}$ is either a cancelling pair-impossible, since $v$ is cyclically freely reduced - or a sister-set. Therefore $l(v) \cdot f(v)$ is a chain word, contradicting the assumption that $v$ is a cyclic geodesic.

So if $c$ contains at least one pair, the arrangement of chains touching $\delta \in\{\alpha, \beta\}$ must either be of the form "Down-Up" or "Down-Partial", or there is one magic corner and no (distinct) chain at the other end. Therefore if $c$ has at least one pair, there is exactly one chain of type "Down" on $\delta$. Recall that this is true also in the case that $c$ has no pairs.

Since the conjugacy disk has exactly four chains, we can embed it in the Cartesian plane so that each relator square is sent to a $1 \times 1$ square with corners at integer coordinates. In this embedding, parallel edge traversals have labels of the same parity, and perpendicular edge traversals have labels of opposite parity. Along edge paths in the boundary whose labels do not include entire chain subwords, nonzero turning angles alternate in value between $\frac{\pi}{2}$ and $-\frac{\pi}{2}$. So if the sign of the first nonzero turning angle along such an edge path is known, then the orientations of the pair of edge traversals of this turning angle determines the precise shape of the edge path in the plane. For example, say we have such an edge path with the first nonzero turning angle positive, and so that the first letter of the pair labels an edge traversal oriented to the right and the second letter of the pair labels an edge traversal oriented upward. Then all the edge traversals along this geodesic path whose labels have the same parity of the first letter of the pair are oriented to the right, and all the edge traversals along this geodesic path whose labels have the opposite parity are oriented upwards.

Corollary 4.7. Assume the situation of Theorem 4.5. When the conjugacy disk is interpreted as a square tiling in the Euclidean plane, the edge paths $\gamma_{o}$ and $\gamma_{t}$ are linear transformations of one another. Therefore, the displacement vector from origin to terminus of $\alpha$ is the same as the corresponding displacement vector for $\beta$.

It follows that the conjugate words $u$ and $w$ have corresponding numbers of letters of the two parities. Also, c contains no pseudo-pairs (any two-letter subword is either a pair or a sister-set).

Proof. Within a disk which is a tiling of relator squares, all edges with traversal labels of the same parity are parallel to each other, and two edges with traversal labels of opposite parity are perpendicular. So corresponding edges of $\gamma_{o}$ and $\gamma_{t}$ are parallel. If $c$ has no pairs, we are done; so suppose $c$ contains at least one pair. It suffices to show that the sign of the first non-zero turning angle along $\gamma_{o}$ is opposite to the sign of the first non-zero turning angle along $\gamma_{t}$. 
We know that $\alpha$ either begins or ends (but not both, unless the links of a a single chain include all of $\alpha$ ) with a chain of type "Down." If necessary, flip the conjugacy disk so that the chain is at the beginning of $\alpha$. We map the disk to the Cartesian plane in such a manner that the first edge traversal of $\alpha$ starts at the origin and goes in the $\hat{\imath}$ direction, and the first edge traversal of $\gamma_{o}$ is in the $\hat{\jmath}$ direction. Note that in reading along $\alpha \circ \gamma_{t}$, the first letter which fails to form a sister-set with the preceding letter labels an edge traversal in the $\hat{\jmath}$ direction.

If the origin of $\alpha$ is not a magic corner, then the first non-zero turning angle along $\gamma_{o}$ must be negative. There is a chain of type either "Up" or "Partial" at the terminus of $\alpha$, so the first non-zero turning angle along $\gamma_{t}$ is positive.

If the origin of $\alpha$ is a magic corner, then the first non-zero turning angle along $\gamma_{o}$ is positive. No disjoint chain touches the terminus of $\alpha$, so the edge path $\alpha \circ \gamma_{t}$ (labelled by the word $w \cdot c$ ) never traces two consecutive non-zero turning angles of the same sign. So all of the edge traversals along this edge path are oriented in either the $\hat{\imath}$ direction or the $\hat{\jmath}$ direction.

We introduce some notation: let $i$ be the parity of the first letter of $w$, and $j$ the other parity. Along the edge path $\alpha \circ \gamma_{t}$, vectors of letters of parity $i$ are all oriented in the $\hat{\imath}$ direction, and all the vectors of letters of parity $j$ are oriented in the $\hat{\jmath}$ direction. If a letter of parity $i$ is followed by a letter of parity $j$, the turning angle between them is positive; if a letter of parity $j$ is followed by a letter of parity $i$, the turning angle between them is negative.

Since the origin of $\alpha$ is a magic corner, the word $c$ must start with letters of parity $j$, followed by a letter of parity $i$.

Therefore the first non-zero turning angle along $\gamma_{t}$ must be negative (a letter of parity $j$ is followed by a letter of parity $i$ ).

Since all of the turning angles of the disk belong to the set $\left\{+\frac{\pi}{2}, 0,-\frac{\pi}{2}\right\}$, any two-letter subword of $c$ must be a pair, sister-set, or pseudo-pair; because of parity and the definition of pseudo-pair, these three possibilities are mutually exclusive. A two-letter word labelling a turning angle of $+\frac{\pi}{2}$ is a pair, one labelling a turning angle of 0 is a sister-set, and one labelling a turning angle of $-\frac{\pi}{2}$ is either a pair or a pseudo-pair (not both).

If a two-letter subword labels the edge traversals of a turning angle of $-\frac{\pi}{2}$ along $\gamma_{o}$, then it necessarily labels a turning angle of $+\frac{\pi}{2}$ along $\gamma_{t}$. A pseudo-pair cannot label a turning angle of $+\frac{\pi}{2}$.

So specifying that two cyclic geodesic words are conjugate but not cyclically equal places considerable restrictions on their form. What happens if two words are cyclically equal? It follows from the geodesic completion theorem [J2] that equal geodesics (in an alternating $C^{\prime \prime}(4)-\mathrm{T}(4)$ presentation with parity and all relators of length four) have identical sign patterns, and corresponding numbers of letters of each parity. We are led to make the following definition:

In the special case that a cyclic geodesic word has even length and its letters alternate in sign, we define its cyclic profile to be a file containing its length and the number of letters of a particular parity. Otherwise, let the cyclic segments of a cyclic geodesic word be maximal subwords which alternate in sign. Then the cyclic profile is a cyclic sequence of files, where each file corresponds to a cyclic segment and consists of 1) the length of the segment, 2) the sign of the first letter of the segment, and 3) the number of letters in the segment of a particular parity. 
Corollary 4.8. In an alternating $C^{\prime \prime}(4)-T(4)$ presentation with parity and all relators of length four, two cyclic geodesics which are conjugate have the same cyclic profile.

Corollary 4.9. If $u$ and $w$ are conjugate (cyclically unequal) cyclic geodesics in a $C^{\prime \prime}(4)-T(4)$ presentation with parity and all relators of length four, then there are cyclic permutations $\tilde{u}$ and $\tilde{w}$ of $w$ and $u$ and a word $d$ which is composed entirely of letters of a single parity so that $\tilde{w} d \tilde{u}^{-1} d^{-1}$ labels the boundary of a disk.

Proof. Start with the situation in Corollary 4.7. As we did in that proof, we may assume that $\alpha$ begins with a chain of type "Down", and that the disk is placed in the Cartesian plane so that the first edge traversal of $\alpha$ starts at the origin and goes in the $\hat{\imath}$ direction, and the first edge traversal of $\gamma_{o}$ is in the $\hat{\jmath}$ direction. We want to choose our new conjugating word so that it labels an edge path which starts at the origin and has all of its edge traversals in the $\hat{\jmath}$ direction.

Suppose that there is a positive turning angle along $\gamma_{o}$. Then for each of the four edge paths $\alpha, \beta, \gamma_{o}$, and $\gamma_{t}$, each edge traveral is either in the $\hat{\imath}$ direction or the $\hat{\jmath}$ direction, and all four corners are in the (closed) first quadrant. Because there is a positive turning angle along $\gamma_{o}$, the corner of the diagram between $\gamma_{o}$ and $\beta$ has a positive $x$-coordinate. Since $\gamma_{o}$ and $\gamma_{t}$ are merely translation images of each other, we may place an identical copy of the diagram in the plane, with corresponding edge paths $\alpha^{\prime}, \beta^{\prime}, \gamma_{o}^{\prime}$, and $\gamma_{t}^{\prime}$, so that $\gamma_{t}^{\prime}$ is identified with $\gamma_{o}$. We claim that the resulting diagram, with boundary edge path $\left(\alpha^{\prime} \circ \alpha\right) \circ \gamma_{t} \circ\left(\beta^{\prime} \circ \beta\right)^{-1} \circ\left(\gamma_{o}^{\prime}\right)^{-1}$, is reduced. This follows because $c$ is geodesic and contains at least one pair, and therefore uniquely determines a tiling within the dimensions specified by the number of letters of each parity. That the diagram is reduced outside of this rectangle follows from the fact that $u$ and $w$ are cyclic geodesics.

If the corner of the new diagram between $\gamma_{o}^{\prime}$ and $\beta^{\prime}$ still has a positive $x$ coordinate, continue to place successive copies of the diagram so that $\gamma_{t}^{(i+1)}$ is identified with $\gamma_{o}^{(i)}$; stop when the corner between $\gamma_{o}^{(n)}$ and $\beta^{(n)}$ has a nonpositive $x$-coordinate. We now have a reduced diagram which has boundary cycle label $w^{n+1} c\left(u^{n+1}\right)^{-1} c^{-1}$. Let $\lambda_{o}$ be the edge path in the diagram that starts at the origin and moves in the $\hat{\jmath}$ direction until it reaches the far side of the diagram. Let $\tilde{\beta}$ start at the terminal vertex of $\delta_{o}$, and travel along $\beta^{(n)} \circ \beta^{(n-1)}$ for the number of edges which is the number of letters in $w$ (hence in $u$ ); the label of $\tilde{\beta}$ is a cyclic permutation of $u$. Let $\lambda_{t}$ be the edge path in the diagram that starts at the terminus of $\alpha$ and moves in the $\hat{\jmath}$ direction until it reaches the far side of the diagram (i.e., the terminus of $\tilde{\beta}$ ). By construction, the label of $\lambda_{t}$ is identical to the label of $\lambda_{o}$. This label we call $d$. (Note that by continuing this building process we could start the new edge path $\lambda_{o}$ at any point along $\alpha^{\prime}$, as long as we start $\lambda_{t}$ further along $\alpha^{\prime} \circ \alpha$ at distance which is exactly the length of $w$.)

If there is a negative turning angle along $\gamma_{o}$, we carry out a similar procedure, only this time the copies of the diagram are placed so that $\gamma_{o}^{(i+1)}$ is identified with $\gamma_{t}^{(i)}$ until the corner between $\beta^{(n)}$ and $\gamma_{t}^{(n)}$ has a nonnegative $x$-coordinate.

So we may assume that the conjugating word has letters all of one parity.

Therefore if $u$ and $w$ are conjugate cyclic geodesics and not cyclically equal, then there is a reduced disk with boundary edge path $\alpha \circ \gamma_{t} \circ(\beta)^{-1} \circ\left(\gamma_{o}\right)^{-1}$ (where the label of $\alpha$ is $w$, the label of $\beta$ is a cyclic permutation of $u$, and the labels of $\gamma_{o}$ and $\gamma_{t}$ are identical) which we may interpret as a tiling of squares in the plane, and 
when we do so the edge paths $\gamma_{o}$ and $\gamma_{t}$ are parallel straight lines. By the remarks preceding Lemma 4.3, each of $\alpha$ and $\beta$ has at least one magic corner, and at the other end a chain either in position "Up" or "Partial" (the second corner is also magic if and only if the tiling is a rectangle). Since the displacement along $\alpha$ equals the displacement along $\beta$, the four corners of the diagram determine a parallelogram. A magic corner necessarily corresponds to an angle of the parallelogram which is not obtuse. So there are two corners of the diagram, diametrically opposed, which are both magic corners. Either the tiling is a rectangle (all the corners are magic), or each of the remaining two corners meets exactly one chain, which is either of type "Up" or "Partial" (the two need not be the same).

Finally, suppose that $u$ and $w$ are cyclic geodesics which are cyclically equalthat is, that a cyclic permutation $\tilde{w}$ of $w$ is equal to a cyclic permutation $\tilde{u}$ of $u$. So $\tilde{u}$ and $\tilde{w}$ label edge paths in the geodesic completion of $\tilde{w}$ with common initial and terminal vertices. There is therefore a subdiagram $D$ with boundary label $\tilde{w} \tilde{u}^{-1}$; this diagram is a reduced diagram and also a square tiling which we may visualize as lying in the Cartesian plane (it is not necessarily a disk). Say that $\tilde{w} \equiv w_{t} \cdot w_{i}$, where $w \equiv w_{i} \cdot w_{t}\left(w_{i}\right.$ and $w_{t}$ are subwords of $\left.w\right)$. On the segment of the boundary of $D$ labelled by $\tilde{w}$, identify the vertex $v$ immediately after $w_{i}$ and before $w_{t}$. Cut the diagram into two pieces by separating along an edge path starting at $v$ and labelled by letters all of one parity, either in the $\hat{\imath}$ direction or the $\hat{\jmath}$ direction (if you like, choose the parity opposite to the first letter of $w$ ). Rearrange the pieces of the diagram in the plane so that the vertex which was formerly the terminal vertex of $D$ is identified with that which was formerly the initial vertex. In this fashion we produce a conjugacy diagram between $w$ and some cyclic permutation of $u$ which is a tiling with straight parallel sides labelled by the conjugating word (which may be trivial).

Corollary 4.10. If $u$ and $w$ are conjugate cyclic geodesics in a $C^{\prime \prime}(4)-T(4)$ presentation with parity and all relators of length four, then there is a cyclic permutation $\tilde{u}$ of $u$ and a word $c$ composedly entirely of letters of a single parity so that there is a diagram with boundary cycle label $c^{-1} w c \tilde{u}^{-1}$ which is a square tiling, with the two portions of the boundary edge path labelled by $c$ being parallel straight lines. If $\tilde{u}$ and $w$ are not cyclically equal, the diagram is a disk, and either the tiling is a rectangle (all the corners are magic), or there are diametrically opposed magic corners and each of the remaining two corners meet exactly one chain, which is either of type "Up" or "Partial" (the two need not be the same).

\section{Conjugacy Algorithm}

We may assume that we are given two cyclic geodesic words $u$ and $w$ of the same even length, both alternating in sign, with corresponding numbers of letters of each parity. If they are conjugate there is a diagram as described in Corollary 4.10; we only have to decide whether such a diagram exists. What we will do is construct all such diagrams, and show that the time required for doing so is actually linear.

First consider the case that $w$ (and therefore $u$ ) has letters of both parities. If $w$ labels an edge path with at least one positive turning angle in the boundary of some square tiling, then $w$ determines the tiling within the dimensions set by the number of letters of each parity that occur in $w$ (i.e, the tiling must agree with the geodesic completion of $w$ where the two diagram would overlap). In the type of diagram described in Corollary 4.10 the edge path labelled by $w$ has a chain of type 
"Down" along it, and therefore has a positive turning angle. We therefore start our construction by taking the square tiling determined by $w$ (the geodesic completion). This tiling may or may not completely fill a rectangle whose dimensions are given by the number of letters of the two parities that occur in $w$. For simplicity, let the first letter of $w$ be oriented in the $\hat{\imath}$ direction, and let the geodesic completion diagram lie in the closed first quadrant; let the terminal vertex of the edge path $\alpha$ labelled by $w$ lie at $(I, J)$. There are two possibilities for a diagram as described in Corollary 4.10: it could lie above $\alpha$, with the edge traversals of the conjugating sides oriented in the $\hat{\jmath}$ direction, or it could lie below $\alpha$, with the edge traversals of the conjugating sides oriented in the $-\hat{\jmath}$ direction.

Any diagram as in Corollary 4.10 lying above $\alpha$ must necessarily have a conjugating word having a common prefix with the word $l_{1}$ which labels the line from the origin along the positive $y$-axis up to (at most) $(0, J)$. More precisely, the conjugating word $c$ could be a subword of $l_{1}$, or $l_{1}$ could be a subword of $c$. (If $l_{1}$ is trivial, there are no conjugacy diagrams lying above $\alpha$.) Similarly if a conjugating diagram were to lie below $\alpha$, the conjugating word would have to overlap the word $r_{1}$ starting at $(I, J)$ and moving in the $-\hat{\jmath}$ direction. So the geodesic completion gives us the letters which must begin any conjugating word which consists entirely of letters of the parity opposite to the first letter of $w$.

What use can we make of this information? If $l_{1}$ is nontrivial, place a copy of $l_{1}$ in the plane starting at $(I, J)$ and moving in the $\hat{\jmath}$ direction. The word $w \cdot l_{1}$ is a geodesic (else $w$ is not a cyclic geodesic), so we extend our current diagram by taking its geodesic completion (i.e, tile so as to fill the resulting corner and across the top of the geodesic completion of $w$ insofar as is possible). The desired conjugacy diagram, if it exists and has $\alpha$ across the bottom, must overlap the diagram we are building. If the additional tiling we have just carried out has created labelled edges on the $y$-axis above $(0, J)$, let the word beyond this point be $l_{2}$. Repeat by placing a copy of $l_{2}$ at $(I, 2 J)$ and continue tiling. As long as the tiling process causes new labelled edges on the $y$-axis, copy these on the right-hand side and continue tiling.

Of course, an analogous procedure applies for diagrams lying below $\alpha$ : place a copy of $r_{1}$ at the origin in the $-\hat{\jmath}$ direction, tile as much as possible, read off $r_{2}$ starting at $(I, 0)$ and moving in the $-\hat{\jmath}$ direction, etc.

This process creates a column with the two vertical sides labelled by the same word (although offset by $(I, J)$ ). Each maximal subdiagram within an $I \times J$ rectangle with one side along the $y$-axis is the geodesic completion of a word conjugate to $w$ via a conjugating word with letters all of one parity. This is illustrated in Figure 3.

We continue this process until the tiling cannot continue or until the diagram starts to repeat $I \times J$ blocks.

We remark that by Corollaries 4.9 and 4.10 in the last section, if $u$ is a cyclic geodesic conjugate to $w$ then the geodesic completion of some cyclic permutation $\tilde{u}$ of $u$ must occur as one of the $I \times J$ subdiagrams - the choice of parity for the conjugating word is unimportant, as well as the particular cyclic permutation of $w$. In fact this now becomes clear on a pictorial level. Note that because the labels of the two vertical sides are identical, offset copies of the column can be placed side-by-side in a diagonal tiling. Consider the resulting diagram (Figure 4).

The construction we have described of the column of tiles is finite and completely deterministic. (It is not obviously polynomial time, but the balance of the paper will show that it is in fact linear time.) When the tiling is complete, the resulting 


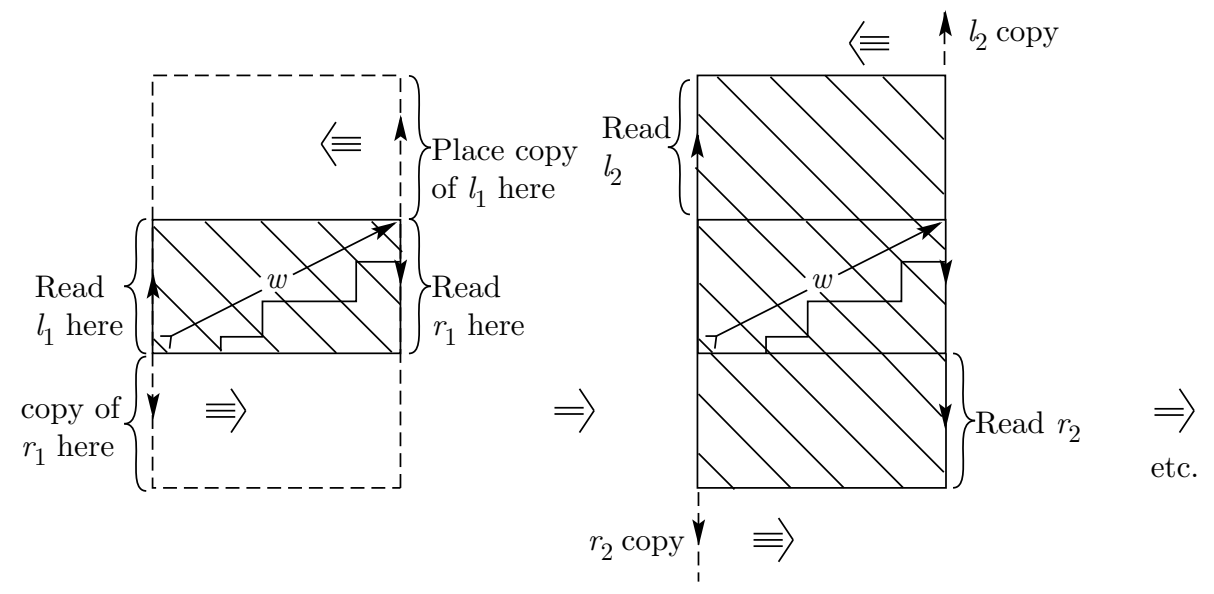

Figure 3

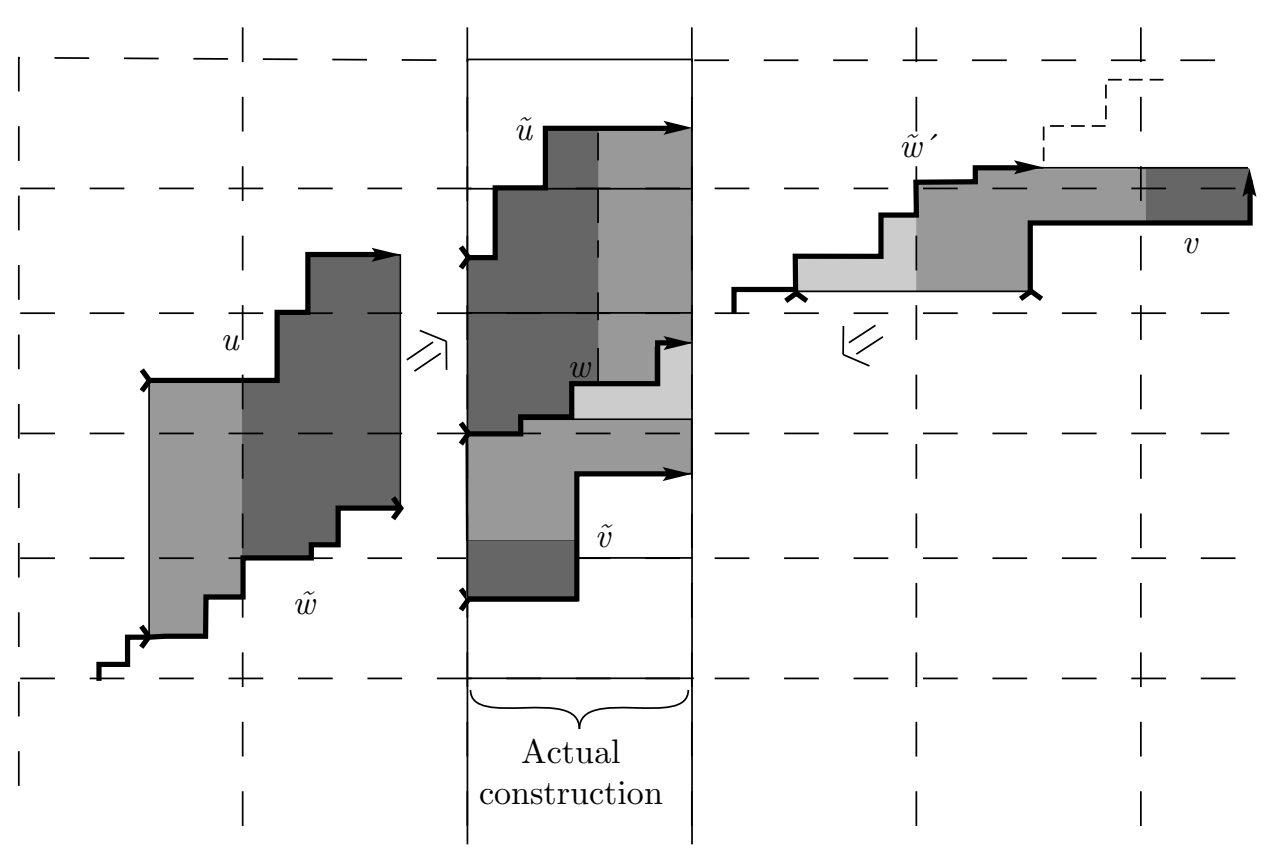

Figure 4

diagram can be searched for cyclic permutations of $u$. If there are $K$ vertices on the left side of the diagram and the length of $u$ is $n$, this search requires up to $K n^{2}$ time: at each of the $K$ vertices, check whether this is the initial vertex of an edge path (reading in $\hat{\imath}$ and $\hat{\jmath}$ directions only) labelled by one of the $n$ cyclic permutations of $u$.

Now consider the case that $w$ is composed entirely of letters of one parity. We cannot use quite the same approach as above, because the square tiling determined 


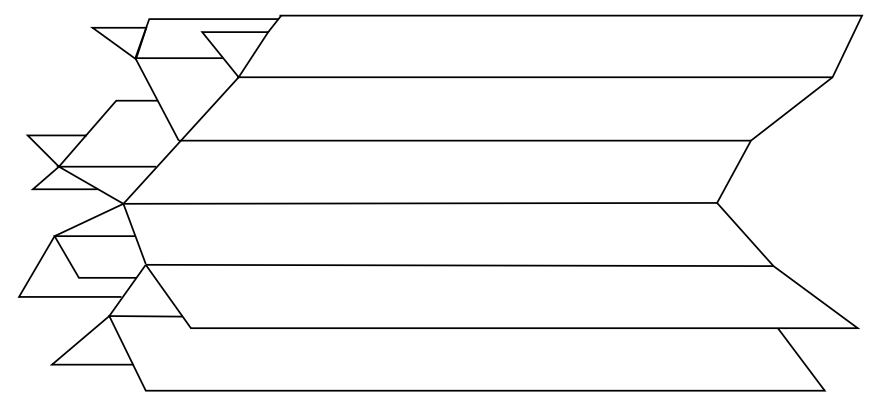

FiguRE 5

by $w$ contains no relator squares (it is just a straight line). So we look for a letter $x_{a}^{\epsilon}$ such that the word $x_{a}^{\epsilon} \cdot w \cdot x_{a}^{-\epsilon}$ is a chain word.

For any such chain, read the word on the new side of the chain, and continue the process from there. That is, for each new word $v=x_{b}^{\epsilon} \cdot w \cdot x_{b}^{-\epsilon}$, look for $x_{c} \not \equiv x_{b}$ so that $x_{c}^{-\epsilon} \cdot v \cdot x_{c}^{\epsilon}$ is a chain word; and so on. This collection of chains gives us a 2-complex which conceivably has the form of a (locally finite) tree under cross product with an interval (Figure 5).

We don't include a chain if the word along the new side is just a cyclic permutation of a word we've already had. This process is finite, because there are only finitely many alternating words of the same length (and parity) of $w$. The words which label the sides of the chains are the cylic geodesics conjugate to $w$ via a word all of a single parity. Search these for cyclic permutations of $u$. If there are $K$ such words and the length of $u$ is $n$, it takes $K n^{2}$ time to search.

This solves the conjugacy problem (cf. [LS]). However, we would like to show that this 2-complex is actually contained in the plane (so that the instantaneous growth is not exponential). We also have to show, in both cases, that the total vertical height of the produced tiling is bounded by a polynomial. (In fact, we will bound it by a constant.)

\section{2-COMPLEX OF CIRCULAR CHAINS IS PLANAR}

We will show in this section that in a 2-complex as described above, at most two distinct chains share a side (so the complex is actually a planar tiling). However, many of the lemmas we develop along the way will then be put to use bounding the growth of our tilings within the plane.

This argument rests upon the geometric origins of the group presentation in the link diagram.

6A. Chains and strolls. Recall that a chain is a reduced disk which is a $1 \times n$ rectangle of 2-cells (see Figure 2). The length of the chain is $n$. We call the two words of length $n$ labelling the (inner and outer) link paths the sides of the chain.

We are particularly interested in chains such that the two clasps are labelled by the same letter, and such that the sides are cyclically freely reduced. (Since the presentation is alternating, this means that $n$ is an even number.) Let us define a chain having such a form to be a circular chain. A cyclic permutation of the relator squares of a circular chain is still a circular chain. We shall consider all cyclic permutations of a circular chain as equivalent to one another. 
Our first step is to characterize [circular] chains in terms of the link diagram. Specifically, we shall show that chains correspond to special kinds of region paths (analogous to edge paths) in the link diagram.

A black (resp. white) parity stroll in the link diagram is a finite sequence $r_{0} C_{1} r_{1} C_{2}$ $\ldots C_{m} r_{m}$, where the $r_{i}$ are regions of black [resp. white] parity such that $r_{i} \neq r_{i+1}$, and $C_{i-1}$ and $C_{i}$ are distinct vertices in the boundary of region $r_{i}$. The length of the stroll is $m$.

Note that a stroll may be thought of as an equivalence class of (topological) paths in the plane of the link diagram. Specifically, we say that a path in the plane of the link projection is a representative path of the stroll if it has initial point in $r_{0}$, terminal point in $r_{m}$, intersects the link projection only and exactly at the crossings $C_{1}, C_{2}, \ldots, C_{m}$, which it encounters in that order, and is composed of $m+1$ simple paths: for $i=1, \ldots, m-1$, there is a simple subpath in region $r_{i}$ between $C_{i}$ and $C_{i+1}$, and for $i \in\{0, m\}$, there is a simple path in $r_{i}$ between the end of the representative path and the crossing point on its boundary. We will often represent a stroll pictorially by a representative path, and blur the distinction between them by referring to a stroll in terms of some representative path.

A stroll of even length $m$ such that $r_{0}=r_{m}$ and $C_{1} \neq C_{m}$ is called a meander. We consider a meander to be a cyclic sequence however, so $r_{0} C_{1} r_{1} \ldots C_{m} r_{0}=$ $r_{i} C_{i+1} r_{i+1} \ldots C_{m} r_{0} C_{1} \ldots C_{i} r_{i}$.

The Correspondence Lemma 6.1. There is a one-to-one correspondence between chains and strolls of equal length, and between circular chains and meanders.

6B. Reading the sides of a stroll. Since a stroll contains precisely the same information as its corresponding chain, it should be possible to read from a representative path of the stroll what the sides of the corresponding chain are. (We shall therefore refer to these words as the sides of the stroll itself.) To do this we will have to examine the link diagram more closely.

Since the link is alternating and the link universe is connected, successive crossings encountered along the boundary of a region $x_{a}$ are all of the same orientation sign relative to $x_{a}$. That is, the oriented angle between the two edges, from the overcrossing strand to the undercrossing strand, is either always positive or always negative for a given region. So it makes sense to refer to the orientation sign of a region.

Since the link universe is connected, it is easy to see that if a region $x_{a}$ is positively oriented, then all regions of the same parity as $x_{a}$ are positively oriented, and all regions of the opposite parity are negatively oriented.

Lemma 6.2. Consider a representative path of an black (resp. white) parity stroll in the link diagram, and specifically a crossing point $v$ along the path marking the transition from one region $x_{a}$ to a region $x_{c}$ (which is of the same parity as $x_{a}$ ). If the regions in question are negatively oriented, then the region of white (resp. black) parity which lies to the left of the crossing point $v$ occurs with positive sign on one side of the stroll's corresponding chain, and the region to the right of the crossing point $v$ occurs with negative sign on the other side of the stroll's corresponding chain. If the crossings are positively oriented with respect to their regions, then the region of white (resp. black) parity which lies to the left of the crossing point occurs with negative sign on one side of the corresponding chain, and that to the right occurs with positive sign. 

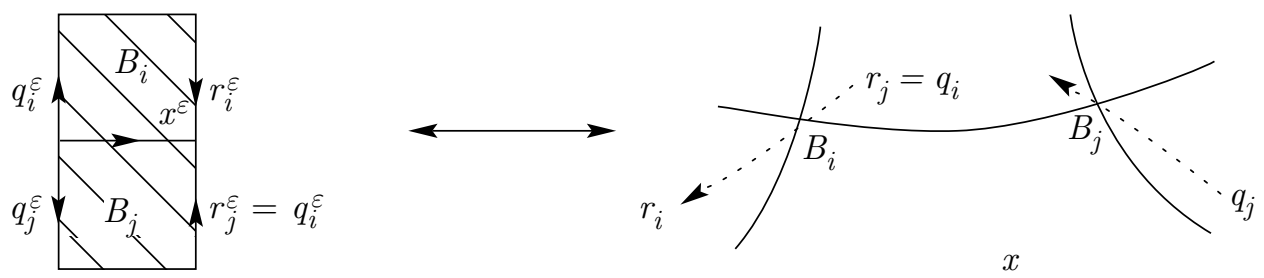

Figure 6

Proof. See Figure 1 and the definition of the Dehn presentation.

Theorem 6.3. The sides of a chain are read from any representative path of the corresponding stroll thusly: if the (unsigned) label of the most-recently-read letter along a side of a chain was that of the region lying to the left of the crossing most recently encountered by the representative path, then the next letter takes its (unsigned) label from the region lying to the right of the next crossing encountered by the representative path; and conversely.

The sign of each letter is as given in the last lemma.

Proof. Follows immediately from the lemma, since a side of a chain must alternate in sign.

Suppose that there are two strolls of length one giving rise to chains with a common side (i.e., to two relator squares with a common side). Then either the strolls are identical, or corresponding regions (and crossings) of the two strolls are distinct. It follows that if two strolls give rise to chains having a common side AND the strolls have a corresponding common region (or crossing), then the strolls are identical.

The corresponding statement in terms of chains is that no pair is a piece; therefore a chain prefix uniquely determines the chain.

So corresponding crossings must be different. This does not imply that the same crossing cannot occur along both strolls, albeit in different positions. A special case is as below:

Lemma 6.4. Given inequivalent strolls $q_{i} B_{i} r_{i}$ and $q_{j} B_{j} r_{j}$ with a common side $x^{\epsilon}$, then $r_{j}=q_{i}$ if and only if vertices $B_{i}$ and $B_{j}$ are distinct endpoints of an edge incident on region $x$ (see Figure 6).

Proof. Vertex $B_{i}$ is the point where regions $q_{i}$ and $r_{i}$ touch their neighboring region $x$ and each other. If $q_{i}=r_{j}$, then $B_{i}$ touches $r_{j}$ as well as $r_{i}$. So $B_{i}$ and $B_{j}$ are distinct vertices on the boundary of neighboring regions $x$ and $r_{j}$, and thus they are the endpoints of one edge. Conversely, if vertices $B_{i}$ and $B_{j}$ are the endpoints of an edge incident on region $x$, then some two of the regions $r_{i}, r_{j}, q_{i}$, and $q_{j}$ are the same. The only way this can happen is if either $q_{i}=r_{j}$ or $q_{j}=r_{i}$.

6C. Snarls and tangles. Recall that we would like to show that there are at most two distinct meanders whose corresponding circular chains have a particular side in common. We will use the fact that the projection is elementary: four distinct regions meet at any vertex of the link universe, and any two distinct regions share at most one edge. 
Imagine a simple closed polygonal curve in the plane of the link projection image which never passes though any crossing point of the projection image, and such that no node of the polygonal curve intersects the projection image; this curve therefore intersects the projection image exactly $2 n$ times for some $n$. Consider the portion of the link diagram corresponding to one of the two domains of the plane thus created. This is called a tangle of $2 n$ leads; if $n=1$ it is called a snarl. We'll sometimes refer to the tangle in the unbounded domain as being annular.

A snarl which includes a simple curve in the link diagram with both endpoints on the polygonal curve is said to be trivial.

Suppose that there are two distinct edge points lying on the common boundary of two regions of the link universe having opposite parities; there is a polygonal curve as above which intersects the link diagram only at these two points. Since two distinct regions share at most one edge, the two points lie on a single edge of the link universe. Therefore, exactly one of the two corresponding snarls is trivial.

This observation will be used repeatedly henceforth without explicit mention.

6D. Defining regions of tangles. Our goal is to show that there are at most two inequivalent meanders with a given common side. Recall that a meander is a stroll of the form $r_{0} C_{1} r_{1} C_{2} \ldots C_{m} r_{m}$ where $m$ is even, $r_{0}=r_{m}$, and $C_{1} \neq C_{m}$. The concatenation of a meander with itself any number of times is still a meander, of course, and self-concatenation on two meanders having a common side results in two (longer) meanders with a common side (the side itself being simply the original shared side, self-concatenated). This being so, it is sufficient to concentrate on inequivalent strolls with a common side, assumed to be as long as we need, possibly invoking the fact that eventually the two strolls simultaneously repeat crossings.

We will concentrate largely therefore on the case of two inequivalent strolls of length three with a common side. In the discussion that follows, we will be considering this question: what restrictions are imposed on the link diagram by the existence of two such strolls? In other words, what relationship in the link universe do their representative paths bear to one another?

To answer our question, we will first need to understand the effects of following a stroll into an unknown tangle. (This will in practice be further complicated by the fact that apparently distinct crossings in our tangle diagrams may not, in fact, be distinct; so certain "regions" implied by our pictures may not even exist. We always include these possibilities as special cases.)

Let us call the $2 n$ regions lying both inside and outside of the simple closed curve of a tangle of $2 n$ leads the defining regions of the tangle. There are $n$ regions of each parity, not necessarily all distinct.

There are two situations which arise frequently in the arguments we will be using; it is convenient to analyze these once and refer back to the reasoning when necessary.

Situation One: Suppose we have a stroll $q B r C s$, and $r$ is a defining region of a tangle. Where does $C$ lie-or equivalently, which regions' labels give the letters read at $C$ ?

Of course $C$ is a crossing point on the boundary of region $r$, and the letter read to the left [resp. right] at $B$ is different from that read at the right [resp. left] at $C$. Naturally, either of the two crossing points may be one appearing along what is known explicitly to be the boundary of the region $r$ in the known portion of the link diagram; we are more interested in the less obvious possibilities. 


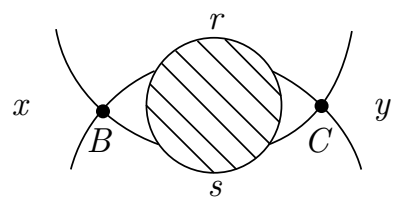

FiguRE 7

Unless the other defining regions of the tangle having the same parity as $r$ are actually known to be distinct from $r$, the crossing $C$ may in fact be a point which does appear explicitly in the known portion of the link diagram, but on the boundary of one of these apparently different regions.

If $C$ is in the tangle proper, it reads on either side a letter labelling a region of parity opposite to $r$, where each region is either 1) a proper subset of the tangle or 2) a defining region of the tangle.

Situation Two: Suppose we have a stroll $q B r C s$, and one of the letters read at $B$ is a defining region of a tangle. Where does $C$ lie-or equivalently, which regions' labels give the letters read at $C$ ?

The question does not assume that the location of $B$ is known. However this question is only of interest if either $B$ is in the tangle or $r$ is a defining region.

Unless the defining regions of the tangle which have the same parity as the region $(x$, say) whose letter is being read at $B$ are actually known to be distinct from $x$, it is possible that $B$ lies not in the tangle or on the known boundary of $x$ at all, but in fact on the boundary of one of these apparently distinct regions.

If $B$ is in the tangle and $r$ is not a defining region, then $r$ is entirely inside the tangle. So $C$ reads on either side a letter labelling a region of parity opposite to $r$, where each region is either 1) a proper subset of the tangle or 2) a defining region of the tangle.

If $r$ is a defining region (whether or not $B$ is in the tangle), then in addition to the possibilities above $C$ can be a point along the boundary of $r$ not in the tangle at all. Keep in mind that a defining region of the same parity as $r$ need not be distinct from $r$, so $C$ can be a point along the boundary of such a region as well.

Remark. Sometimes for greatest generality in the arguments throughout this section, crossings near a tangle which are depicted as being distinct, need not be distinct at all. A common example is shown in Figure 7, where the regions $x$ and $y$ are known to be distinct (note that $r$ and $s$ must be distinct). Such a diagram shall be referred to as a frog.

It may actually be that crossings $B$ and $C$ are the same crossing, that is that the regions $x, y, r$, and $s$ all meet at a common vertex $B=C$. In this case the frog is said to be improper.

6E. Inequivalent strolls on parallel tracks. Now consider two inequivalent strolls $q_{i} B_{i} r_{i} C_{i} s_{i}$, with a common side $x^{\epsilon} y^{-\epsilon}$ (read first from the right, then from the left sides of the strolls, say). Earlier we observed the implication of (for example) $q_{2}=r_{1}$. What is the implication of having the same crossing occur in both of the two strolls - necessarily at different points along the strolls? This is the same as asking, in Figure 8, what the strolls look like if $B_{2}=C_{1}$ (which implies by parity that $q_{2}=r_{1}$ and $\left.r_{2}=s_{1}\right)$ ? 


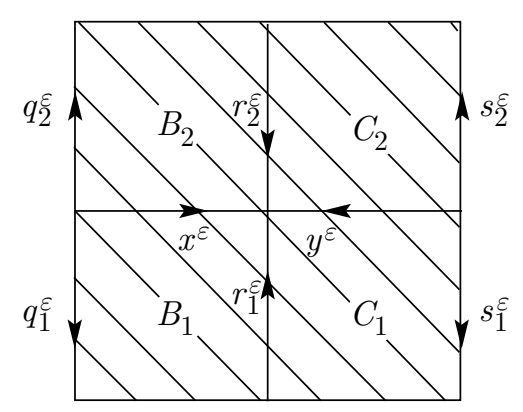

Figure 8

If $B_{2}=C_{1}$, then this crossing is a common crossing point of regions $x$ and $y$ (since $x$ lies to its right and $y$ to its left). So $B_{1} r_{1} C_{1}$ is a path from one common crossing point of neighboring regions $x$ and $r_{1}$ to a different common crossing point; so $B_{1}$ and $C_{1}$ are the endpoints of the common edge separating regions $x$ and $r_{1}$. Similarly $B_{2}\left(=C_{1}\right)$ and $C_{2}$ are the endpoints of the common edge separating regions $y$ and $r_{2}$. (See for example Figure 25, below.)

We will return to this case later. For now, simply note that $B_{1}$ and $B_{2}$ are the two endpoints of an edge incident on $x$, and that $C_{1}$ and $C_{2}$ are the two endpoints of an edge incident on $y$.

Now say that $B_{2}=C_{1}$ and $C_{2}=B_{1}$. So in addition to the above, $C_{2}=B_{1}$ is a common crossing point of regions $x$ and $y$, distinct from $B_{2}=C_{1}$. This is therefore a simple link. (A simple link is an alternating link of two components with two crossings.) Note that any stroll goes back and forth between the two crossing points, so its sides are always both alternating; in fact, any meander of a given parity and length in a simple link is equivalent to any other. So any conjugacy tiling is planar and repeats after a single chain or two, so we don't need to worry about this case.

Now we consider cases where the vertices $B_{1}, C_{1}, B_{2}$, and $C_{2}$ are all distinct. Also, we want to make our claim a little stronger; we will now treat these as beginning strolls of length three with a common side $x^{-\epsilon} y^{\epsilon} z^{-\epsilon}$. We suppose these letters are read first from the right of the strolls, then from the left, and then from the right again. Note that $z$ is a right-hand (from the oriented strolls) neighbor of both $s_{1}$ and $s_{2}$. Now what do we know about the link diagram of Figure 9? Note that we may consider this diagram in terms of a known area buffering an inner tangle of four leads, and an annular tangle of eight leads, as in Figure 10.

Lemma 6.5. We have inequivalent strolls $q_{i} B_{i} r_{i} C_{i} s_{i} D_{i} t_{i}$, for $i \in\{1,2\}$, with a common side $x^{-\epsilon} y^{\epsilon} z^{-\epsilon}$. Suppose the vertices $B_{1}, C_{1}, B_{2}$, and $C_{2}$ are all distinct.

Then either $z=x$, or the vertices $C_{1}$ and $C_{2}$ are distinct endpoints of an edge incident on the region $y$. In the latter case, either $s_{1}=r_{2}$ or $s_{2}=r_{1}$.

Proof. Consider Figure 10. We know that the defining regions of the inner tangle of four leads are all distinct. Apply analysis of Situation Two to stroll one, which enters the inner tangle of four leads: so $z=y$, or $z$ is properly contained in the inner tangle, or $s_{1}$ is actually the defining region $r_{2}$. So we need only consider the case that $z \neq x$ is properly contained in the inner tangle. Apply analysis of Situation One to stroll two: so $z$ is one of the regions $a, b$, or $y$, or entirely outside the circle 


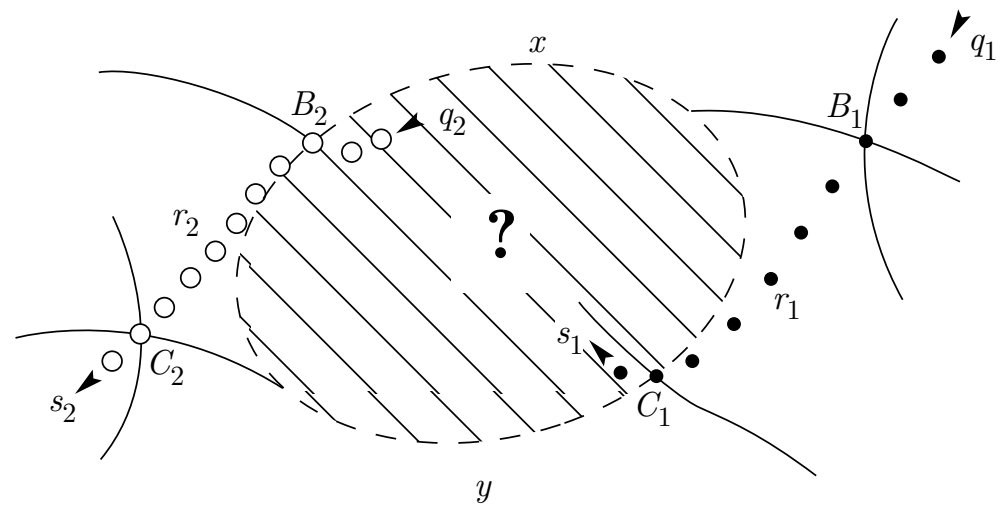

FiguRE 9

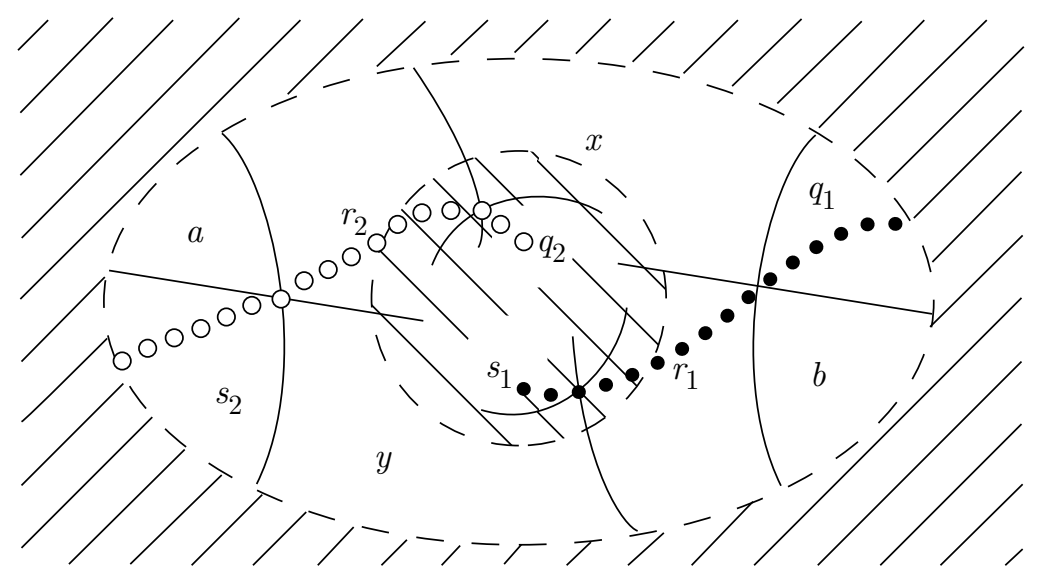

FiguRe 10

of the annular tangle (but none of these is properly inside the inner tangle), or $s_{2}$ is one of $r_{1}, r_{2}$, or $q_{1}$. But $s_{2} \neq r_{2}$, and $q_{1}$ is separated from the region $z$ inside the inner tangle unless $q_{1}=r_{1}$ (which is impossible) or $q_{1}=r_{2}$ (but $r_{2} \neq s_{2}$ ). So the only viable option in this case is that $s_{2}=r_{1}$.

If $s_{i}=r_{j}$, then $C_{i}$ touches $r_{j}$ as well as $r_{i}$. So if $z \neq x$, then $C_{1}$ and $C_{2}$ are distinct points on the boundary of $y$ and $r_{i}$. So they lie on one edge.

Theorem 6.6. Suppose distinct strolls $q_{i} B_{i} r_{i} C_{i} s_{i} D_{i} t_{i}(i \in\{1,2\})$ have a common side of the form $x^{-\epsilon} y^{\epsilon} x^{-\epsilon}$. If vertices $B_{1}$ and $B_{2}$ are endpoints of an edge incident on region $x$, then vertices $C_{1}$ and $C_{2}$ are endpoints of an edge incident on region $y$.

Proof. We may first assume that $C_{i} \neq B_{j}$, or else the statement has already been shown. Consider the two possible pictures, as shown in Figure 11.

In the left-hand figure (case $q_{1}=r_{2}$ ), there is a true tangle with defining regions $x, r_{1}, y$, and $r_{2}$. The second stroll has to read $x$ at its next vertex $D_{2}$, so either $s_{2}=r_{2}$ (impossible) or $s_{2}=r_{1}$, which implies that $C_{1}$ and $C_{2}$ are endpoints of an edge incident on $y$. In the right-hand figure we use the same reasoning. In this case, it is easy to see that the tangle between $x$ and $y$ includes a (proper) frog from $B_{2}$ to $C_{1}$. 


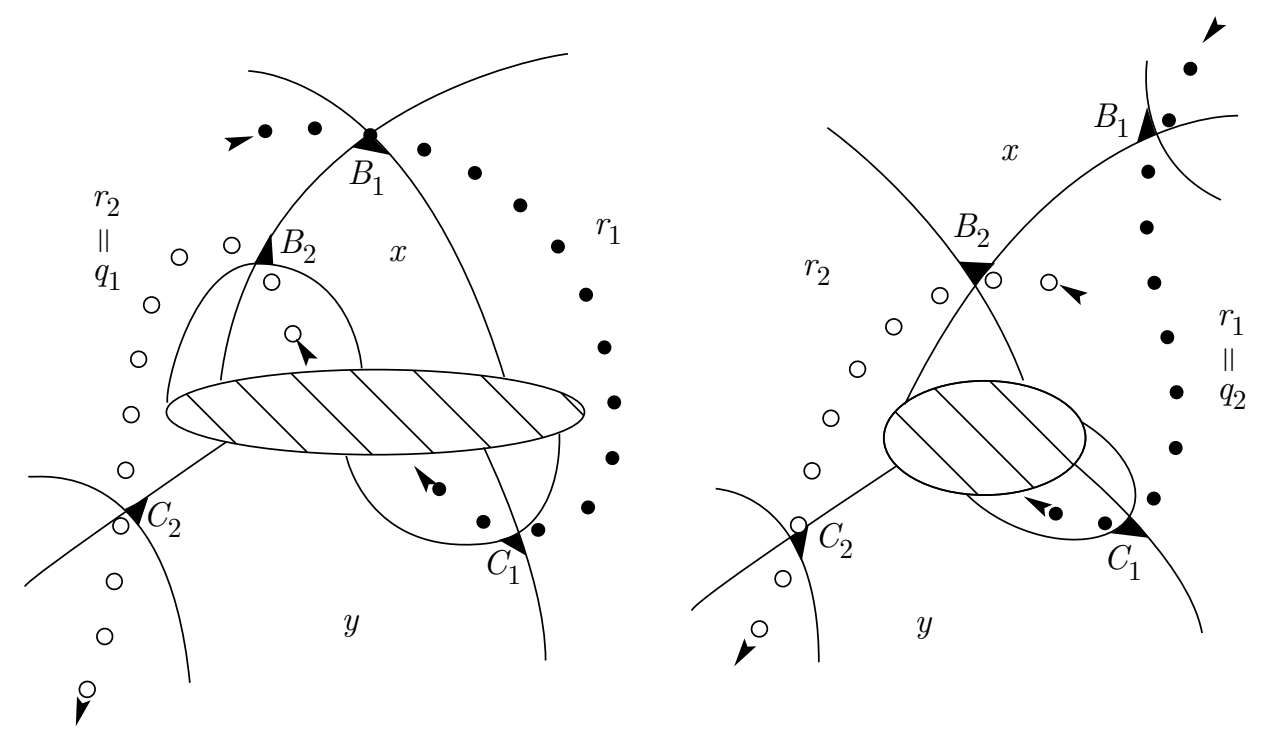

FIGURE 11

Suppose we have two inequivalent strolls $r_{0} C_{1} r_{1} \ldots C_{k} r_{k}$ and $s_{0} D_{1} s_{1} \ldots D_{k} s_{k}$ reading a common side $y_{1}^{\epsilon} y_{2}^{-\epsilon} \ldots y_{k}^{\delta}$. We say that the strolls run on parallel tracks if for $j=2$ to $k-1$ the vertices $C_{j}$ and $D_{j}$ are distinct endpoints of an edge incident on region $y_{j}$. Our goal is to show that inequivalent strolls with a common side either run on parallel tracks, or else the common side alternates back and forth between just two letters.

To this end we now consider two inequivalent strolls of length four, corresponding to chains of length four with a common side. Formally, these strolls are $p_{i} A_{i} q_{i} B_{i} r_{i} C_{i} s_{i} D_{i} t_{i}$, for $i \in\{1,2\}$, where the corresponding chains of each have a side $w^{\epsilon} x^{-\epsilon} y^{\epsilon} z^{-\epsilon}$. We suppose these letters are read first from the left of the strolls, then from the right, then from the left and right again.

Lemma 6.7. Let distinct strolls with a common side having a subword of the form $x^{\epsilon} y^{-\epsilon} x^{\epsilon} z^{-\epsilon}$ be given. Then either $z=y$, or the link diagram has a subdiagram as in Figure 12 (where the frog may be improper). In the latter case, the strolls run on parallel tracks.

Proof. The two strolls necessarily read $y^{-\epsilon}$ at distinct crossings on the boundary of $y$. The situation is therefore as below shown in Figure 13 .

None of the tangles shown need be true: $b$ could be $x, c$ likewise, $k$ could be $l$, and (for example) $n$ could be $j$. By symmetry, it doesn't matter in which direction the strolls are being read; so let us suppose that it is from right to left.

So Situation One occurs when the left-hand stroll enters the outermost annular tangle $\tau_{o}$ of eight leads. We see that $z$ must be properly inside the outermost tangle $\tau_{o}$, or $d$ or $a$ or $y$, or (if $n=j$ ) $b$ or properly inside $\tau_{b}$.

Now Situation Two applies to the right-hand stroll at the central tangle $\tau$. So $z$ must be properly inside $\tau$, or $y$, or (if the three regions $k, l$, and $x$ have a common crossing) $c$ or properly inside $\tau_{c}$ or (if instead $k=l$ and if the three regions $l, x$, 


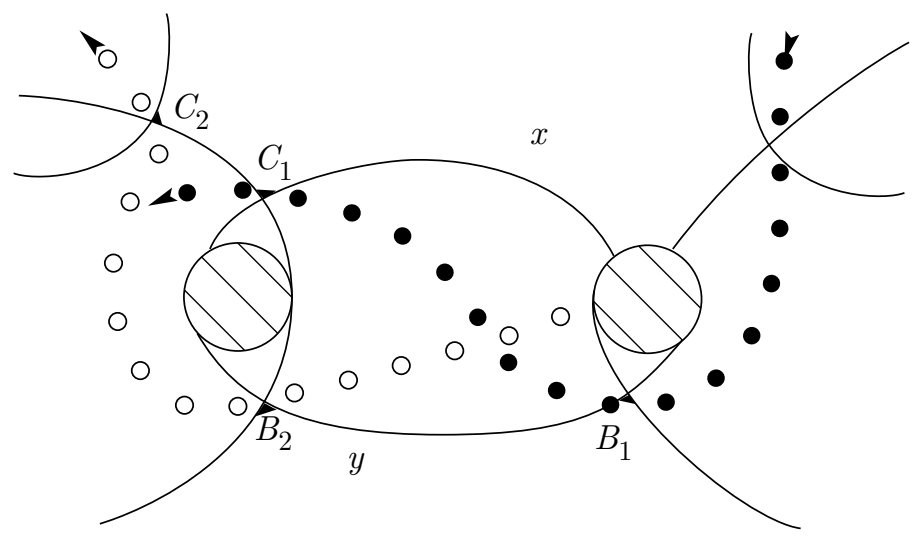

FIGURE 12

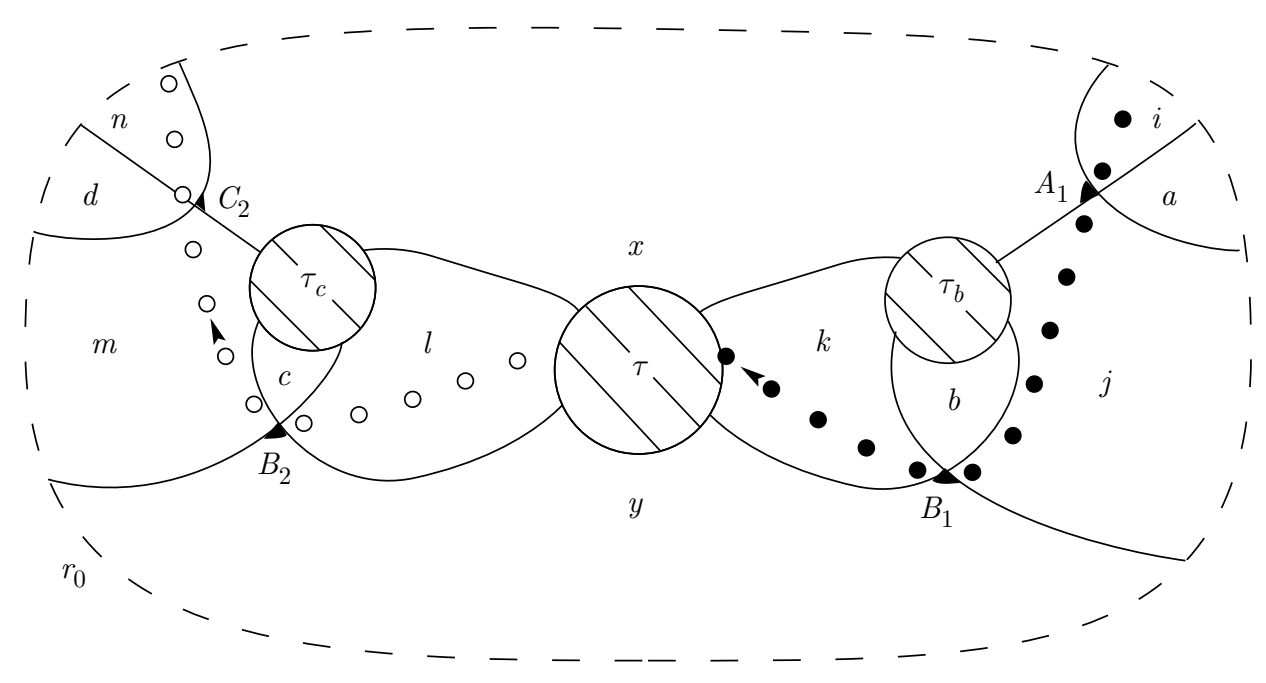

FIGURE 13

and $m$ have a common vertex) be properly inside the outermost tangle $\tau_{o}$, or $d$ or $a$, or (if additionally $m=j$ ) $b$ or properly inside $\tau_{b}$.

Thus we must either have that $z=y$, or that $k=l$ and the regions $l, x$, and $m$ have a common vertex, as claimed.

Roughly speaking, when the common side of two inequivalent strolls starts alternating between two letters, it tends to continue alternating between those lettersor else there is a frog involved, and the strolls run on parallel tracks.

The Parallel Tracks Theorem 6.8. Suppose that two inequivalent strolls share a side. If a single pair of corresponding vertices are distinct endpoints of an edge incident on the region whose letter is being read, then the strolls run on parallel tracks. If the strolls do not run on parallel tracks, then the common side is formed entirely of alternations between two letters. 


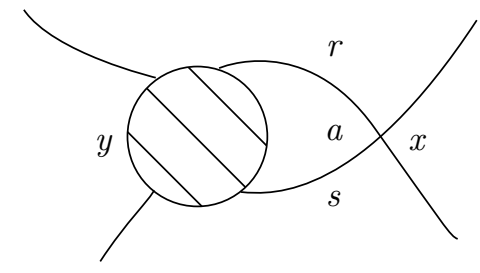

FIGURE 14

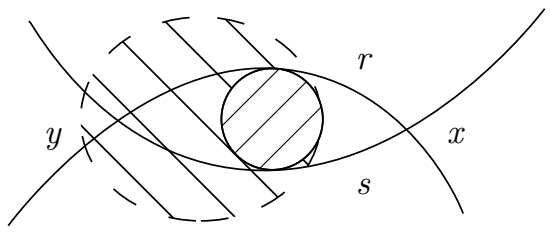

FiguRE 15

Proof. We begin by considering strolls of length three $q_{i} B_{i} r_{i} C_{i} s_{i} D_{i} t_{i}, i \in\{1,2\}$, reading $x^{-\epsilon} y^{\epsilon} z^{-\epsilon}$. If we know that $C_{1}$ and $C_{2}$ are distinct endpoints of an edge incident on region $y$, we're done; this is automatically the case if $z \neq x$. So suppose that $z=x$, and (say) $B_{1}$ and $B_{2}$ are distinct endpoints of an edge incident on region $x$. Then invoke Theorem 6.6 .

Now suppose that the length is greater than three. In any consecutive triple of corresponding pairs of vertices of the two strolls, the middle pair of vertices form the endpoints of an edge, or else the common side has the form $x^{\epsilon} y^{-\epsilon} x^{\epsilon}$ and none of the pairs of vertices form endpoints of an edge. Induct along successive triples. Note that if any subword of the common side has the form $x^{\epsilon} y^{-\epsilon} z^{\epsilon}$ where $z \neq x$, then the strolls must lie on parallel tracks.

6F. Straight words of alternating letters. It is therefore of interest to consider strolls with a side that alternates between two letters. Such a stroll must either eventually pass back through a previous region, or terminate without repeating. The following lemma explores the implications of the first possibility.

Let a subdiagram as in Figure 14 be called a toad; note that the regions $r$ and $s$ must be distinct, because they share a vertex (Figure 15).

The toad is proper if $y \neq a$, improper otherwise. A toad may include a frog, meaning that regions $y, r$, and $s$ have a common vertex.

Note that each frog is included in some toad.

The Helix Lemma 6.9. If there is a stroll with side $\left(x^{\epsilon} y^{-\epsilon}\right)^{n}\left[x^{\epsilon}\right]$ which passes through a region twice, then any representative path of the stroll (together with the repeated region) encircles one of the two regions $x$ or $y$, and the other lies outside this path.

If there exists a meander with side $\left(x^{\epsilon} y^{-\epsilon}\right)^{n}$ then there is a subdiagram of type either A (where the number of toads is even; see Figure 16) or B (where the number of frogs is odd; see Figure 17).

There are at most two inequivalent meanders sharing a given side of the form $\left(x^{\epsilon} y^{-\epsilon}\right)^{n}$. If there are two, we are in the case I.A with all toads including frogs, and at least one of these frogs is proper.

Proof. Assume a stroll has a side which alternates between two letters $x^{\epsilon}$ and $y^{-\epsilon}$, and that it passes through a sequence of regions $r_{0} r_{1} r_{2} \ldots r_{k}=r_{0}$, and crossings $C_{1} C_{2} \ldots C_{k}$. One of the regions of the letters being read, $x$ say, lies always to the right as we traverse a representative path; $y$ lies always to the left. The representative path is contained wholly in the closures of regions having parity opposite to that of $x$ and $y$ (each of which is a connected domain), so the representative path cannot cross either region (see Figure 18). 

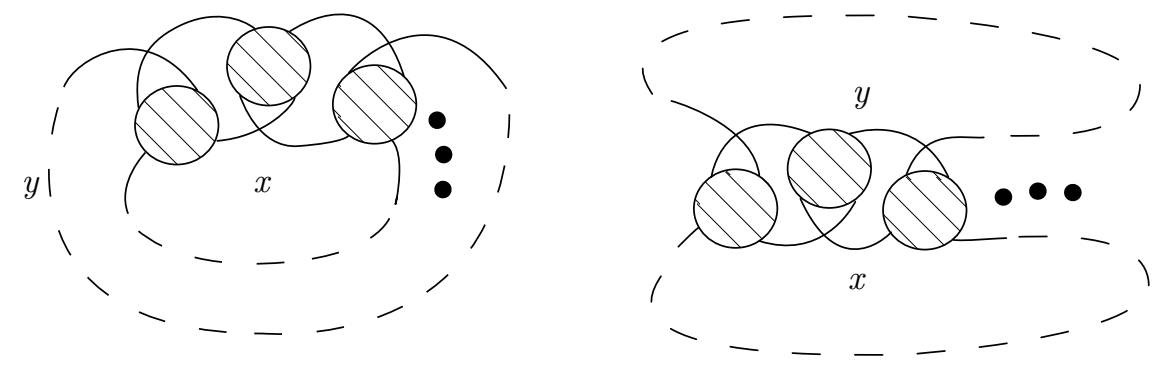

Figure 16. Case A.
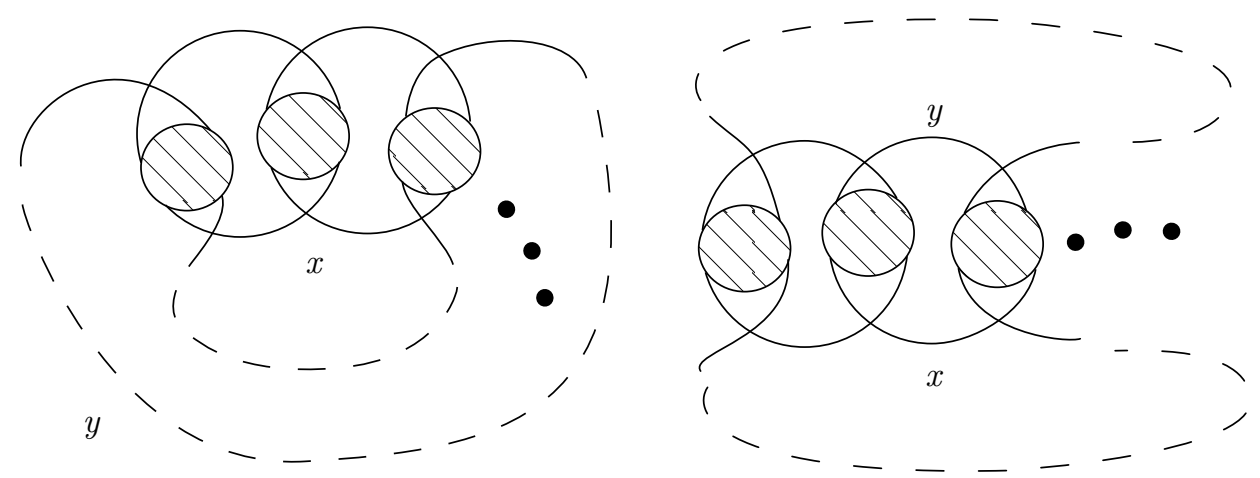

Figure 17. Case B.

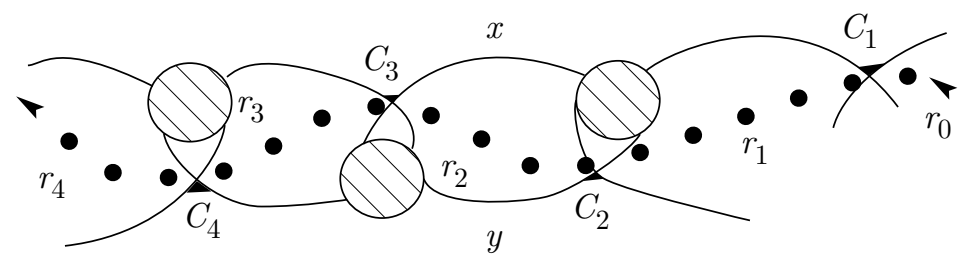

FiguRE 18

If $\alpha$ is the representative path, then $\alpha \cup r_{0}$ must circumnavigate either the region $x$ or the region $y$. One of the regions lies entirely inside the bounded domain of $\mathbb{R}^{2} \backslash\left\{\alpha \cup r_{0}\right\}$, the other entirely in the unbounded domain.

Now suppose that there is a meander with a side alternating between $x^{\epsilon}$ and $y^{-\epsilon}$. Consider a portion of the path with regions and crossings as named above, such that $r_{k}=r_{0}$ is the only repeated region. The last crossing encountered before the repeated region is $C_{k}$ (which necessarily lies on the boundary of $r_{0}=r_{k}$ ); we consider two cases, based on whether $k$ is even or odd. Say that the letter read at $C_{1}$ is $x^{\epsilon}$, and it is read from the right side of the representative path.

If $k$ is even, the letter read at $C_{k}$ is $y^{-\epsilon}$. The region $r_{0}=r_{k}$ has been repeated after an interval of even duration, so $r_{0} C_{1} r_{1} \ldots C_{k} r_{k}$ is itself a meander.

If the given meander has length greater than $k$, the letter it would read at $C_{k+1}$ would be $x^{\epsilon}$. The only crossing incident on $r_{0}=r_{k}$ from which $x^{\epsilon}$ can be read on the correct side is $C_{1}$. Similarly, the meander must continue on, repeating $r_{1} C_{2} r_{2} \ldots$. 


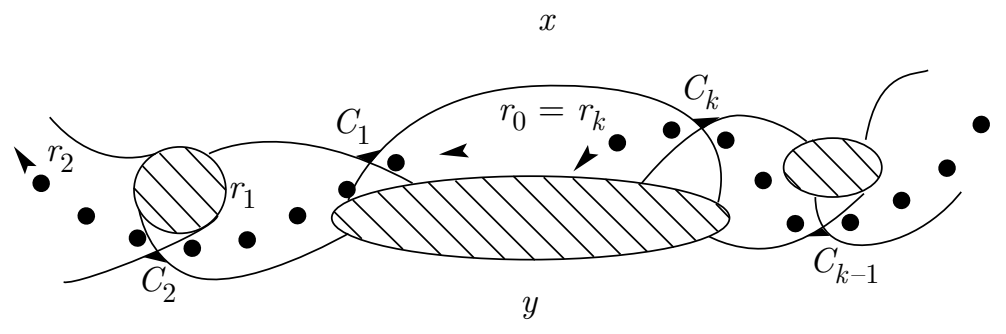

FiguRe 19

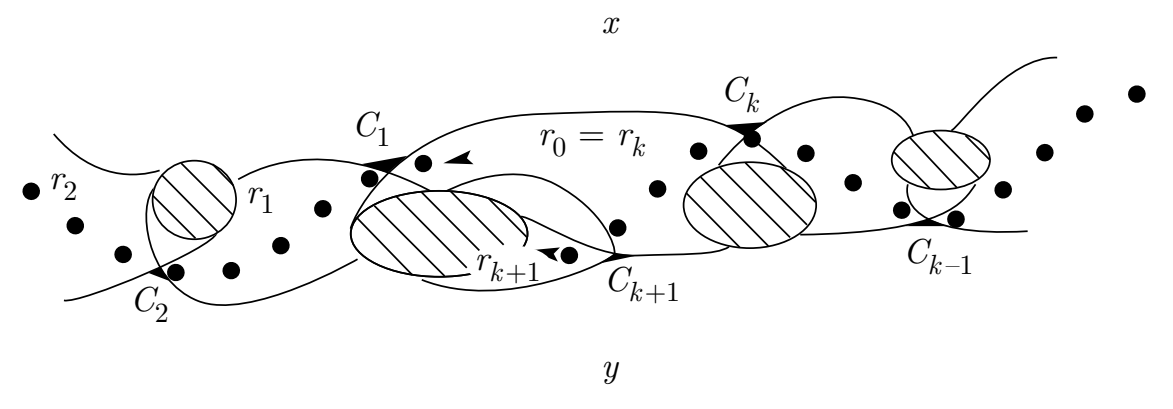

FiguRe 20

So the meander must be an integer multiple of $r_{0} C_{1} r_{1} \ldots C_{k}$, plus a final $r_{0}$. This is case $\mathrm{A}$.

If $k$ is odd, the letter read at $C_{k}$ was $x^{\epsilon}$, so crossings $C_{1}$ and $C_{k}$ are endpoints of an edge between $x$ and $r_{0}=r_{k}$. Note that although $r_{k}=r_{0}$ is a repeated region, because it has appeared after an interval of odd duration we do not have a meander yet. So the meander must continue on, and the next letter it reads (at $C_{k+1}$ ) must be $y^{-\epsilon}$. Therefore the region $r_{0}$ neighbors $y$ (see Figure 19).

Now the meander must still continue, so at $C_{k+2}$ the letter $x^{\epsilon}$ must be read; this implies that regions $r_{k+1}$ and $x$ are neighbors. This is only possible if $r_{k+1}=r_{1}$ (Figure 20).

Because the region $r_{1}$ has been repeated after an interval of odd duration, the meander must keep going, reading $y^{-\epsilon}$ at $C_{k+2}$ and so forth. So each of the pictured toads must include a frog, as the menader passes again through regions $r_{1}$ to $r_{k}=r_{0}$, which this time is repeated after an interval of even duration. So the meander is some integer repetition of $r_{0} C_{1} r_{1} \ldots C_{2 k}$, plus $r_{0}$. This is case $\mathrm{B}$.

So we know that a meander with a side composed entirely of two letters implies that the link diagram has either the form A or B. In a diagram of the type B, any meander with a side alternating between $x^{\delta}$ and $y^{-\delta}$ uses all $k$ vertices incident on region $x$, and all $k$ vertices incident on region $y$. Of course two meanders with a common alternating side have the same length. So in case B, two meanders with a common side of alternating form are cyclic permutations of each other (and are thus equivalent meanders).

Therefore, if there are two distinct meanders with a common alternating side, we must have a diagram of type A. 


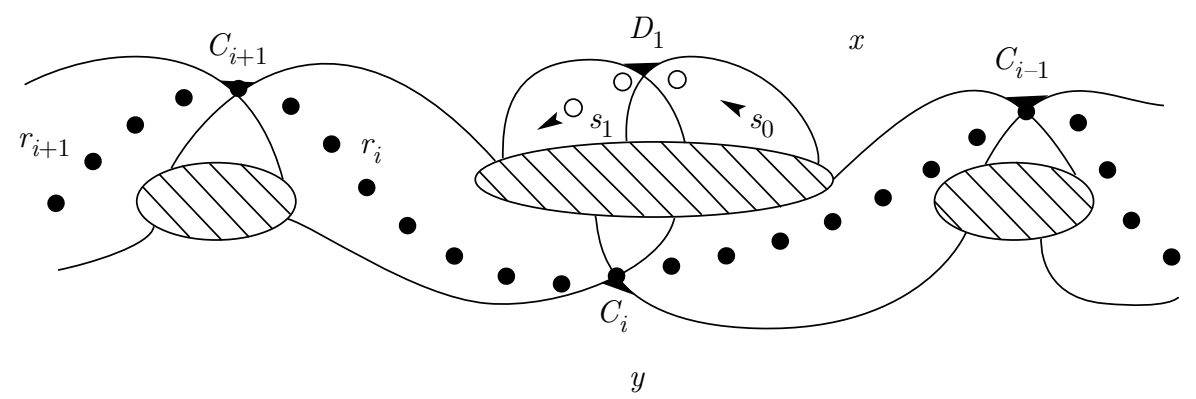

Figure 21

Say we have two such distinct meanders, $r_{0} C_{1} r_{1} \ldots r_{k}$ and $s_{0} D_{1} s_{1} \ldots s_{k}$. It must be that $D_{i} \neq C_{j}$ for any $i=j(\bmod 2)$, else the second meander is simply a cyclic permutation of the first. So we have the situation shown in Figure 21.

But the new meander must read $y^{-\epsilon}$ to its left at its next crossing $D_{2}$, so $s_{1}=$ $r_{i}$; which implies that $s_{2}=r_{i+1}$, and so on. Thus all the toads contain frogs. If all of these frogs are improper, the two meanders are cyclically equivalent, a contradiction.

6G. Tree-complex is planar. After two short lemmas, we will be ready to tackle our basic result, stated below.

A word that is the side of some chain is said to be a straight word. Note that a straight word is freely reduced and consists entirely of sister-sets (specifically, the letters are all of one parity and alternating in sign). The sides of a circular chain are cyclically freely reduced straight words of even length, all of the letters having the same parity. Call a (cyclic) word which forms the side of a circular chain a circular word.

Theorem 6.12. A straight word of length three either labels the side of no more than two inequivalent chains, or has the form $x^{\epsilon} y^{-\epsilon} x^{\epsilon}$. A circular word of the form $\left(x^{\epsilon} y^{-\epsilon}\right)^{n}$ labels the side of no more than two distinct meanders, and occurs only if the link diagram has the form described in the Helix Lemma 6.9.

Lemma 6.10. Suppose distinct strolls $p_{i} A_{i} q_{i} B_{i} r_{i} C_{i} s_{i}$ read a common word of the form $w^{\epsilon} x^{-\epsilon} y^{\epsilon}$, where $w \neq y$. If $C_{1}=B_{2}$, then there are at most two strolls reading the given word.

Proof. We have done the case where $C_{1}=B_{2}$ and $C_{2}=B_{1}$ (this was the case of a simple link). So we may assume that $C_{2} \neq B_{1}$.

Suppose that there is a third stroll $p_{3} A_{3} q_{3} B_{3} r_{3} C_{3} s_{3}$ reading the given word. Then $B_{3}$ is the other endpoint of an edge incident on $x$ with endpoint $B_{1}$, as well as the endpoint of a (necessarily distinct) edge incident on $x$ with endpoint $B_{2}$. A similar relationship holds for $C_{1}, C_{2}$, and $C_{3}$.

It follows that regions $x$ and $y$ both have degree three, and we have the situation shown in Figure 22.

Stroll two can read $w$ only from tangle 1 ; stroll three only from tangle 2 .

Lemma 6.11. There are at most two distinct strolls reading a word $w^{\epsilon} x^{-\epsilon} y^{\epsilon}$, where $w \neq y$. 


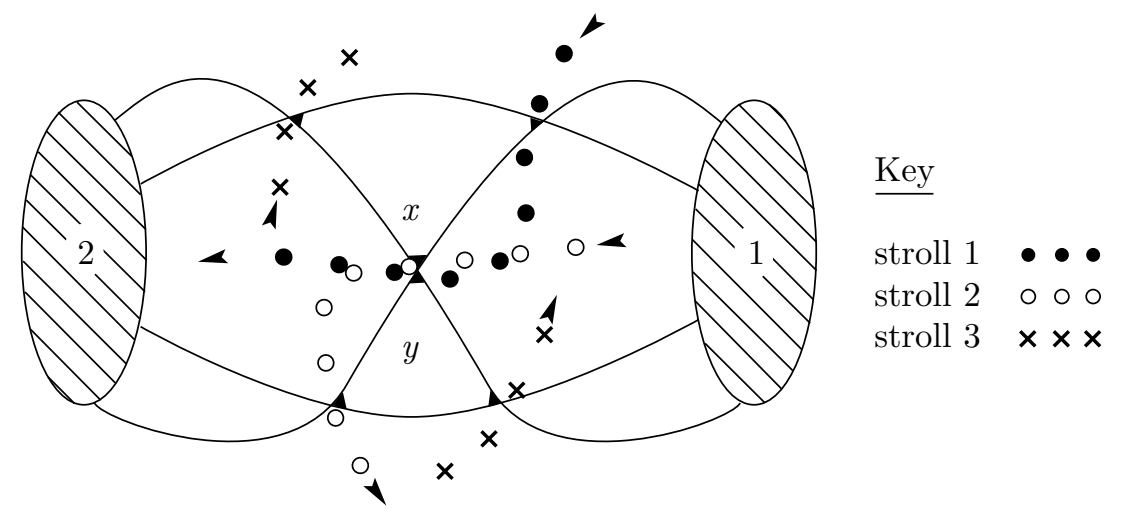

FiguRe 22

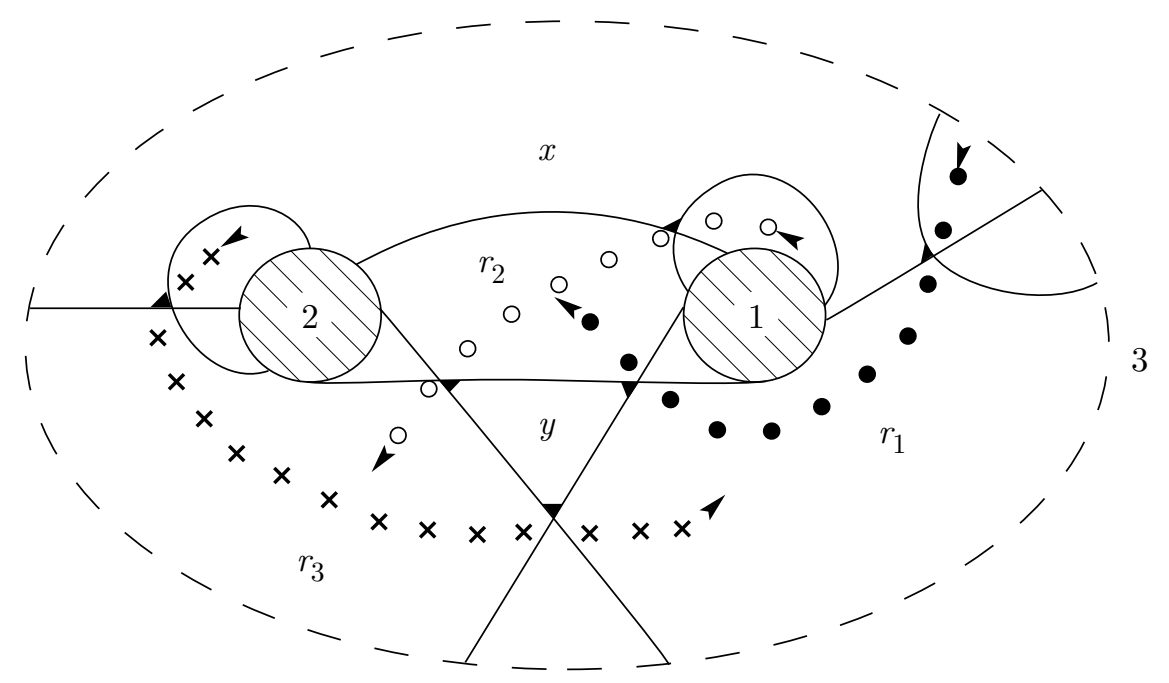

FIGURE 23

Proof. Suppose we have distinct strolls $p_{i} A_{i} q_{i} B_{i} r_{i} C_{i} s_{i}$ reading $w^{\epsilon} x^{-\epsilon} y^{\epsilon}$. In the last lemma we considered the possibility that $C_{1}=B_{2}$. So we may now suppose that $C_{1} \neq B_{2}$. We know that $B_{1}$ and $B_{2}$ are endpoints of an edge incident on region $y$. If there is a third such stroll, then the degree of region $y$ is three, and we have a subdiagram as in Figure 23.

Where does the region $w \neq y$ lie? According to stroll one, $w$ lies in tangle 2 or tangle 3 ; according to stroll two, in tangle 1 or tangle 3 ; according to stroll three, in tangle 1 or 2 . There is no common possibility.

This concludes the proof of Theorem 6.12.

We now know that even if the words we wish to test for conjugacy are straight the process of building up all possible conjugate geodesics using relator squares is a process confined to the plane. So the instantaneous growth of our 2-complex (which 


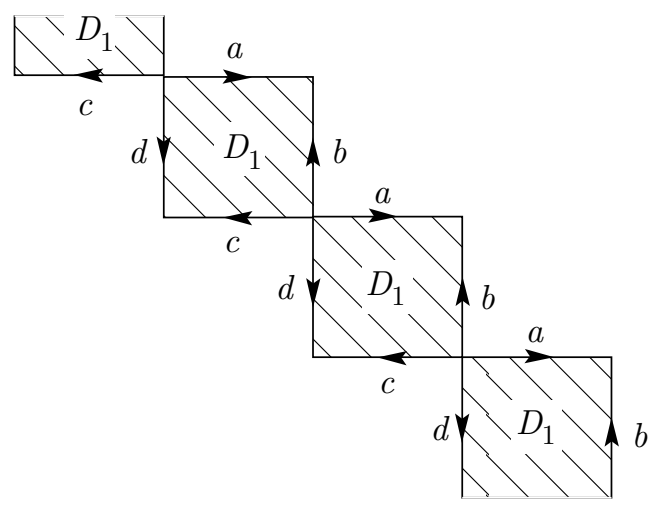

FigURE 24

we now know to be a disk) is polynomial. It remains to bound the total growth of the tiling, i.e., to limit the total number of relator squares used.

\section{Vertical GROWTH is Bounded By a CONSTANT}

7A. The log cabin tiling. Before undertaking this final step, we make a slight digression which will nevertheless prove useful.

Proposition 7.1. If our augmented Dehn presentation is taken from any alternating prime link except an unlink, then there exists a circular chain which tiles the Euclidean plane.

Proof. Choose a crossing $v_{1}$ of the link diagram $K$. Consider the relator square corresponding to this crossing to have some set alignment $D_{1}$ relative to the Cartesian plane. Say that as we trace out the component of $K$ which contains the overcrossing segment of $v_{1}$ in an arbitrary direction, we encounter (not necessarily distinct) crossings $n$ times before returning to our starting point. Let these crossings have the names $v_{i}, i \in\{1, \ldots, n\}$, in the order encountered. (For a knot, each crossing will have two names.)

Place copies of $D_{1}$ all along the diagonal, from upper left to lower right, of an infinite square, as shown in Figure 24.

Note that this tiling produces two kinds of corners, each labelled by a pair; and the pairs in question belong to the relators arising at the crossings adjacent to $v_{1}$ along the chosen component of $K$, namely $v_{2}$ and $v_{n}$. So place the corresponding relator squares down the adjacent diagonals, in alignments $D_{2}$ and $D_{n}$ as appropriate. We can continue this process at any step, because if $x_{e}^{\epsilon} x_{f}^{-\epsilon}$ is a pair produced at one end of the edge separating regions $x_{e}$ and $x_{f}$ in the link diagram, then $x_{f}^{\epsilon} x_{e}^{-\epsilon}$ is a pair produced at the other end (the link is alternating). Of course we may encounter a previous crossing from a new direction, but because we are on a different segment of the crossing, the "color" (i.e., alignment) of the relator squares will be different.

The circular chain $D_{1} D_{2} \ldots D_{n}$ is a repeating subset which tiles the plane.

Because of its similarity to a kind of quilt pattern, we call such a tiling pattern a $\log$ cabin tiling. Note that if we consider any $n \times n$ square subdiagram of the tiling, parallel sides of the square are labelled by identical words. So the above proof also 


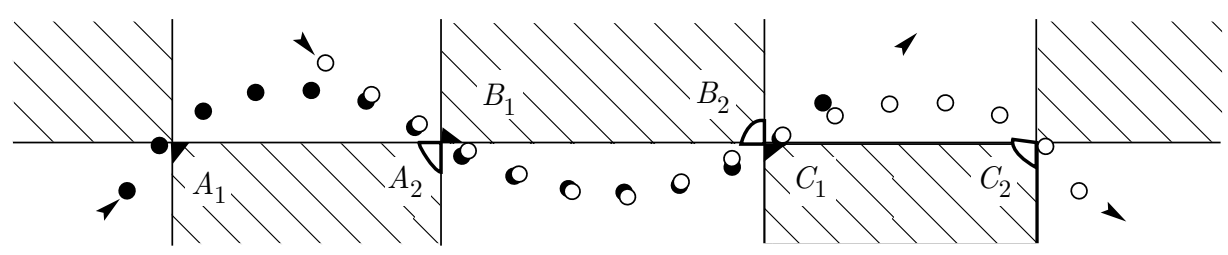

Figure 25

implies that the free abelian group on two letters is a subgroup of the link group (as long as the link is not an unlink); hence, the link group is not negatively curved in the sense of Gromov.

Since the circular chain we used was obtained by making a single complete circuit of a link component, its length is no more than twice the number of link crossings (it is exactly twice the number of crossings if and only if the link is a knot). The circular chain corresponds to a meander which follows alongside a link component, snaking back and forth across the component at crossings in order to stay in regions of the same parity.

Of course the log cabin tiling consists of cyclic permutations of a single circular chain, so if we take consecutive chains in a rectangular subset of the tiling and examine their corresponding strolls, we see that they are identical except for being one region out of phase (see Figure 25).

We say that the second stroll shadows the first, or that the two strolls are one out of phase. Of course, if the strolls continue to the end of the link component, the tiling repeats.

We shall see that shadowing is the most common sort of relationship between strolls with a common side. The basic exception is when there is some straight word that is of the form $\left(x^{\epsilon} y^{-\epsilon}\right)^{n}\left[x^{\epsilon}\right]$.

7B. A constant bound. Let $k$, throughout this section, be the number of crossings of the link diagram. We know that the number of regions is $k+2$ (by Euler's formula for a connected graph); at most $k$ of these regions can be of a given parity, since four distinct regions meet at a vertex, two of each parity. (So there are at least two regions of each parity.)

We know that if we have distinct strolls of length four with a common side, then either corresponding vertices of the strolls form the endpoints of an edge, or else the common side is of the form $\left(x^{\epsilon} y^{-\epsilon}\right)^{2}$. More is true; in the second case, if the two strolls can be extended and still have a common side, then either the maximal length to which the two strolls can be extended is bounded by the number of regions neighboring both $x$ and $y$ (hence by $k$ ); or the strolls are parts of meanders as in the Helix Lemma.

The reason is that as soon as the common side of the two strolls reads three distinct consecutive letters, corresponding vertices are exactly distance one apart; and this relationship propagates down the strolls. So if there is no portion of the link diagram as in the Helix Lemma, then two strolls reading $\left(x^{\epsilon} y^{-\epsilon}\right)^{n}\left[x^{\epsilon}\right]$ must cease to have a common side once all the regions neighboring both $x$ and $y$ have been exhausted.

Up until now we have confined our attention to a pair of strolls with a common side. Now we consider a sequence of four strolls of length four: the first having a 


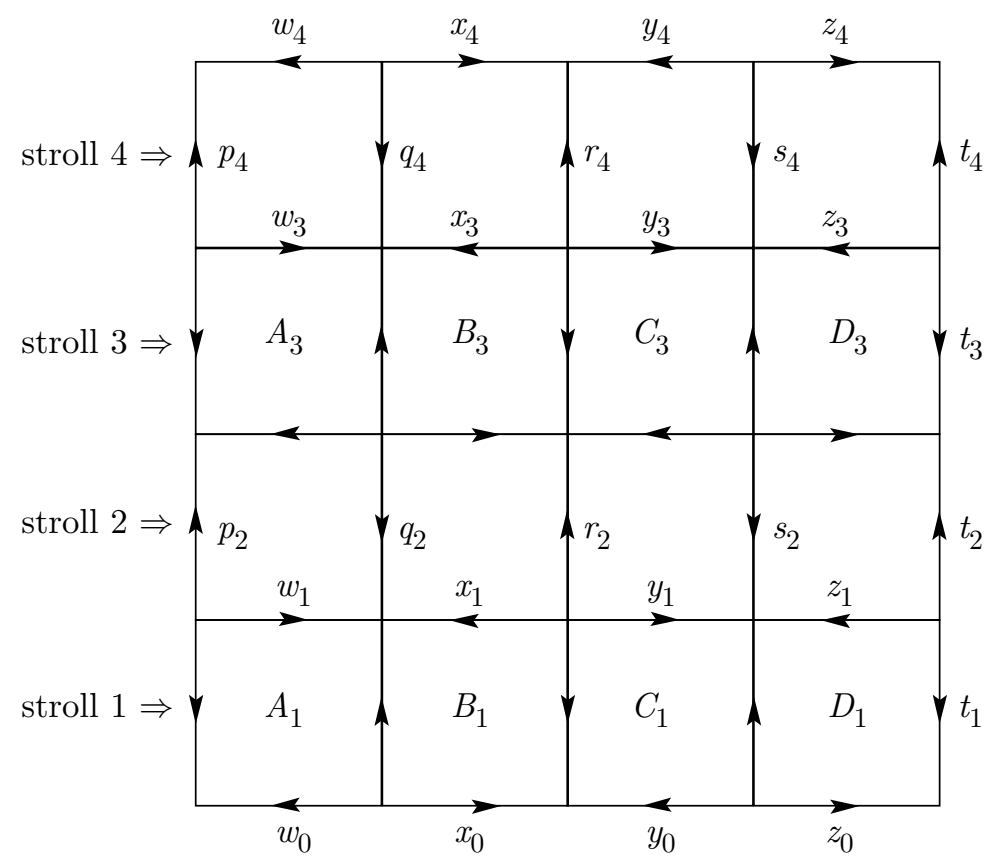

FigURE 26
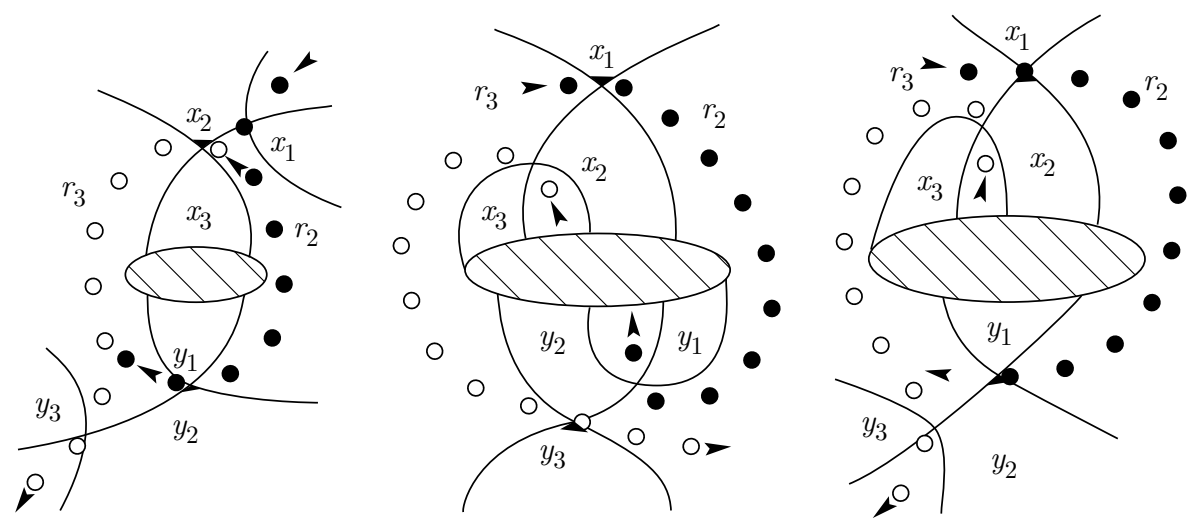

FiguRE 27

common side with the second, the second having a common side with the third, and the third having a common side with the fourth. Let the vector labels and relator squares of the corresponding chains be as named in Figure 26. (Of course, this rectangle also represents a sequence of four strolls of the opposite parity, each having length four.)

Concentrate on the two middle strolls, stroll 2 and stroll 3. Suppose that the middle sections of these two strolls have a relationship of one of the three types shown in Figure 27 (where the frog in the first diagram is proper, i.e., $B_{3} \neq C_{2}$ ).

In each case there is a simple closed curve encircling the frog; this curve passes only through regions $r_{2}$ and $r_{3}$, and vertices $B_{i}$ and $C_{j}$ at the two ends of the frog. 
If a stroll of length two has one crossing incident on a region strictly outside the curve, and the other incident on a region strictly inside the curve, then either one of the vertices is $B_{i}$ or $C_{j}$, or else the middle region of the stroll is $r_{2}$ or $r_{3}$. In each case, regions $x_{3}$ and $y_{1}$ lie strictly inside the curve, and $x_{1}$ and $y_{3}$ lie strictly outside; it is not possible for $B_{1}$ to be $B_{2}$, or for $C_{4}$ to be $C_{3}$. Therefore we must conclude, in such cases, that $r_{1}=r_{3}$ and $r_{4}=r_{2}$.

Suppose the four vertical chains are extended as long as possible. By our earlier analysis, they must either break off before $k$, or else the center vertical line is circular and labelled by a word of the form $\left(r_{1}^{-\epsilon} r_{2}^{\epsilon}\right)^{n}$.

Consider the case of a conjugacy diagram where the word $w$ is composed entirely of letters of one parity. Here each row of the tiling is a circular chain; we stop tiling when the new horizontal word is a cyclic permutation of a word that has already appeared. We may therefore assume that of two successive circular chains, neither is a cyclic permutation of the other. (In particular, the second is not one out of phase with the first.)

Either there is a horizontal circular word of the form $\left(x^{\epsilon} y^{-\epsilon}\right)^{n}$ (which puts us in the case of the Helix Lemma), or between any pair of strolls corresponding to successive chains in the tiling, one of the three diagrams of Figure 27 appears. In the latter case, either the tiling is no deeper than $k$, or else there is a vertical circular word of the form $\left(x^{\epsilon} y^{-\epsilon}\right)^{n}$ (which puts us once again in the Helix Lemma).

Now consider the case of a conjugacy diagram where the word $w$ has letters of both parities. Here consecutive strolls may be one out of phase. If all consecutive pairs of strolls are one out of phase, then the tiling is log cabin and repeats in at most $2 k$ chains. If there is a consecutive pair of strolls related via one of the three diagrams of Figure 27, then, by the same reasoning as before, either the tiling terminates by $k$ or the Helix Lemma is satisfied. Now take the remaining case, that the only consecutive pairs of strolls that are not one out of phase with each other are those with a common word of the form $\left(x^{\epsilon} y^{-\epsilon}\right)^{n}\left[x^{\epsilon}\right]$. In this case it is helpful to regard the tiling as horizontal rather than vertical. If $\left(x^{\epsilon} y^{-\epsilon}\right)^{n}$ is the side of some meander, the Helix Lemma is satisfied; otherwise, the depth (or width if you prefer) is bounded by $k$.

It remains to bound vertical growth in the case of the Helix Lemma.

We have a circular word $\left(x^{\epsilon} y^{-\epsilon}\right)^{n}$, and the link diagram has one of the forms described in the Helix Lemma. It is not necessarily the case that the meanders to either side of the circular word are distinct; they may be copies of a single meander, out of phase with one another by an even number of vertices. Suppose for a moment that all the toads (or frogs) in the diagram are improper; then the two meanders are cyclically equivalent and read a word $\left(x^{\epsilon} y^{-\epsilon}\right)^{n}$ on both sides. If the circular word is horizontal, then the tiling is trivial. If the circular word is vertical, all the vertical chains are just cyclic permutations of this one, and all repeat at the same point, bounded by $2 k$. So we may assume that some toad is proper.

Either the tiling is only two letters across in some dimension (and repeats at or before $2 k$ in the other), or these two chains are part of a sequence of chains.

Lemma 7.2. Suppose we have a sequence of three meanders, the first having a common side with the second, the second having a common side of the form $\left(x^{\epsilon} y^{-\epsilon}\right)^{n}$ with the third. Also suppose that one of the toads described by the Helix Lemma is proper. Then the regions $x$ and $y$ are joined via a sequence of exactly two frogs, and one of the frogs includes a region of degree two touching $y$. 


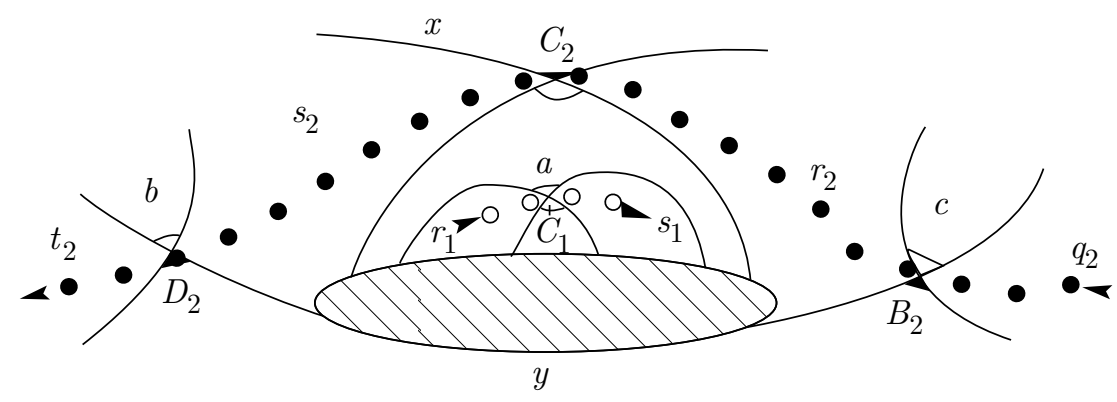

FigURE 28

$I$.

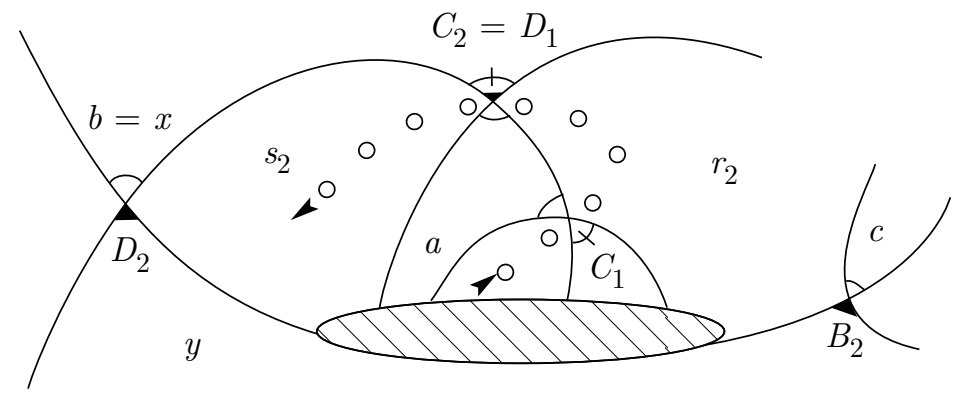

FigURE 29

Proof. Consider meander two as it passes through the proper toad. Let the pertinent substroll be $q_{i} B_{i} r_{i} C_{i} s_{i} D_{i} t_{i}$ where $i$ is the number of the stroll and vertex $C_{2}$ marks the point where region $x$ meets the proper toad (see Figure 28).

Since the toad is proper, the region $a$ is not $y$, so at some vertex $B_{1}$ at distance one from $B_{2}$ stroll one encounters $a$ to its left. So either $r_{1}=s_{2}$ or $s_{1}=r_{2}$ (possibly both).

There is a proper tangle inside a simple closed curve through regions $a, s_{2}, y$, and $r_{2}$. Since stroll one next encounters $b$ (outside the tangle), either $s_{1}=s_{2}$ (impossible) or $s_{1}=r_{2}$. Even so, how can this stroll one possibly encounter $b$ ? There are two ways: first, if $x=b$, we can have the situation shown in Figure 29; or second, if $b \neq x$ is a neighbor of $r_{2}$; but since $b$ touches $y$ (which has only two vertices in common with $r_{2}$ ), this implies that $b=c$, as in Figure 30. In either case, since stroll one encounters $c$ just before encountering $a$, it follows that $r_{1}=s_{2}$. (In case $I$ it further follows that $c=x$.) See Figure 31 .

Note that since meander two then goes back to $a$ again, meander one must do so also, implying that $B_{1}=C_{1}$. Consider the other case for a moment (Figure 32 on page 899).

Meander one started one region further counterclockwise than meander two did, but after reading two letters is running one region behind. Suppose that $B_{2} \neq D_{2}$. If the next toad encountered by meander two is proper and thus a letter other than $y$ is read, meander one just can't make it over to the next crossing to read the new letter. So the next toad is improper and meander two reads $y$ there, forcing 
$I I$.

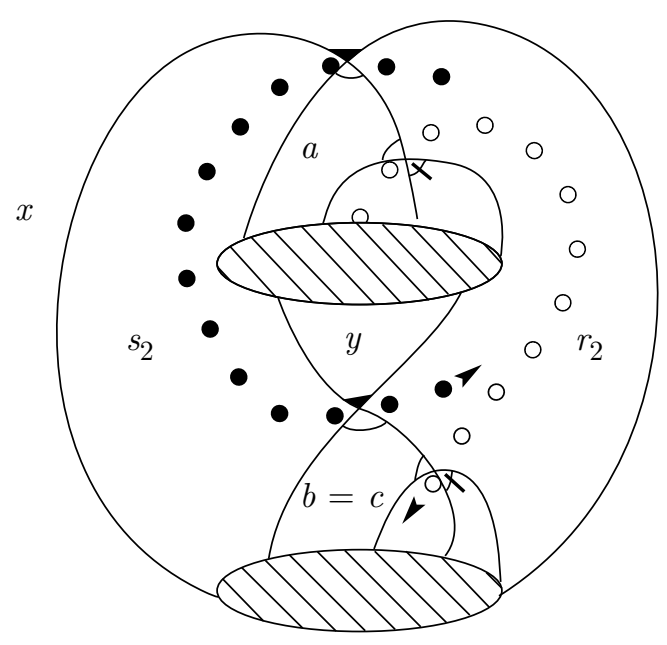

Figure 30

II.

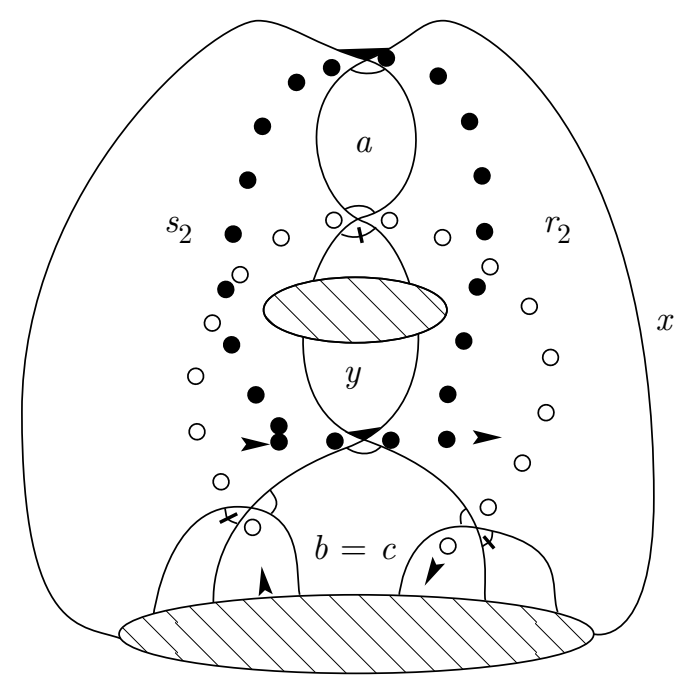

FiguRE 31

meander one to leave region $s_{2}$ via crossing $D_{2}$. Inductively, all of the other toads are improper, and meander one goes all the way around the circuit of improper toads one region behind meander two. But then meander two gets back to $a$ again while meander one is still a region behind and can't get to $a$ ! The only way for the two meanders to have common sides is if the total number of toads is two, i.e., if $B_{2}=D_{2}$. This diagram is thus as shown in Figure 33 on page 899 .

So in both case $I$ and case $I I$ the number of toads is exactly two. In both cases the region $a$ was seen to be of degree two. 
$I$.

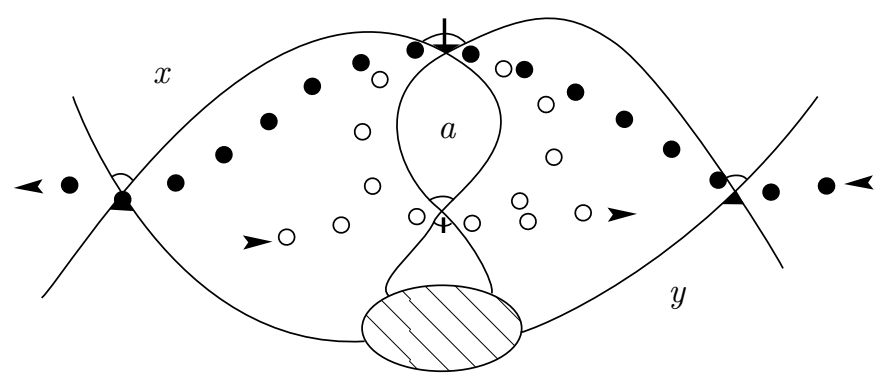

FiguRE 32

$I$.

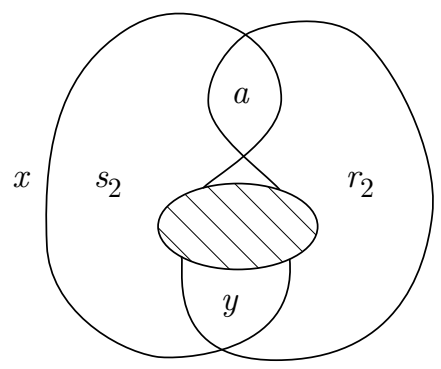

FiguRE 33

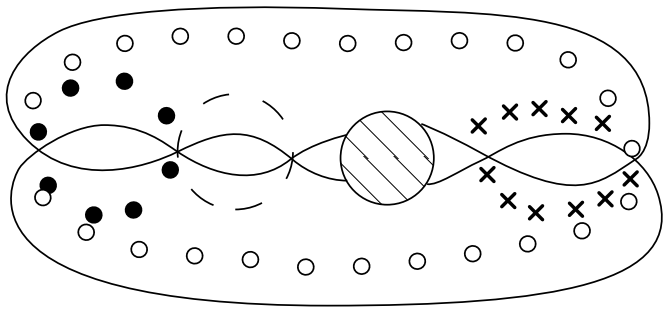

FiguRE 34

Since the number of toads is exactly two, it is not possible for meander three to be a copy of meander two out of phase. So both toads include frogs.

Consider case $I$ of the last lemma. Under what conditions can the sequence of chains be extended to four? (Otherwise we have a $3 \times 2$ block only.) It is not difficult to see that the diagram must have the form shown in Figure 34.

So if we continue to stack meanders, each must be of length two, and the total number of such meanders is bounded by the number of regions of degree two neighboring both $x$ and $y$, and thus by $k$. (Note that such a sequence has a unique possible sequential order.)

Now consider case $I I$. We notice that vertices $B_{i}$ and $C_{i}$ are not endpoints of an edge. This implies that there is a word in our tiling, perpendicular to the circular 
word $\left(x^{\epsilon} y^{-\epsilon}\right)^{n}$, of the form $\left(r^{\epsilon} s^{-\epsilon}\right)^{m}\left[r^{\epsilon}\right]$, which either terminates before $k$ or is a circular word.

If $\left(r^{\epsilon} s^{-\epsilon}\right)^{m}$ is a circular word, we know that for its chain to appear as the middle chain in a sequence of three there must be exactly two frogs running between regions $r$ and $s$; but our diagram implies that there are at least four. So in this case the height is bounded either by two (if the word $\left(x^{\epsilon} y^{-\epsilon}\right)^{n}$ is vertical) or by $2 k$ (if the word $\left(r^{\epsilon} s^{-\epsilon}\right)^{m}$ is vertical, since the width is just two).

If on the other hand there is no circular word $\left(r^{\epsilon} s^{-\epsilon}\right)^{m}$, then if the word $\left(x^{\epsilon} y^{-\epsilon}\right)^{n}$ is horizontal we're done. So suppose that it's vertical, putting us necessarily in the case that the horizontal rows of the conjugacy diagram are non-circular and shorter than $k$. In this case, simply regard the tiling as horizontal instead of vertical. The tiling is no deeper than $k$.

\section{Polynomial time}

Suppose any word of length $n$, we can replace it by an equivalent geodesic in $\zeta n^{2}$ time: start to embed an initial segment of the word in $\mathbb{E}^{2}$ using the standard embedding (delete any cancelling pairs $x^{\epsilon} x^{-\epsilon}$ as they occur). When a pair is read, place the appropriate relator square as well, and as long as the chain prefix continues, continue to place the appropriate relator squares. As soon as a letter is read which completes a chain word, delete the chain from the diagram, letting the chain word be replaced by the inner link path label of the chain. Continue this process to the end of the word. The total number of tiles required is less than $n^{2}$.

It follows that given any word of length $n$, we can replace it by a conjugate cyclic geodesic in $\zeta n^{2}$ time; do the above, treating the word as cyclic and tiling on the surface of a cylinder (of appropriate variable radius).

Given two cyclic geodesics, one can check whether they have the same cyclic profile in $c n$ time, where $n$ is the length of the shorter.

Given two cyclic geodesics with the same cyclic profile, length $n$, but not alternating as cyclic words, one can check whether they are cyclically equivalent in $\zeta n^{2}$ time (if not, they are not conjugate). (Take the geodesic completion of the tiling obtained above for one of the words, and check whether the other word - or a cyclic permutation of its cyclic segments - labels a path-homotopic geodesic edge path.)

Given two cyclic geodesics with the same cyclic profile, of even length $n$ and alternating in sign, we determine whether they are conjugate (including the possibility that they are cyclically equivalent) by laying the appropriate tile for the geodesic completion of one of the words, and searching it for all cyclic permutations of the other. The tiling takes time $2 k \zeta n$. The search takes time $n \times 2 k n$ time.

Polynomial Time Theorem 8.1. The computation time for determining whether two cyclic geodesics are conjugate is bounded by a cubic polynomial. The computation time for determining whether two arbitrary words are conjugate is bounded by a polynomial of degree five.

\section{REFERENCES}

[A] K. I. Appel, On the Conjugacy Problem for Knot Groups, Math. Z. 138 (1974), 273-294. MR 50:10090

[AS1] K. I. Appel and P. E. Schupp, The Conjugacy Problem for the Group of any Tame Alternating Knot is Solvable, Proc. Amer. Math. Soc. 33 (1972), 329-336. MR 45:3530 
[AS2] _ Artin Groups and Infinite Coxeter Groups, Invent. Math. 72 (1983), 201-220. MR 84h:20028

[D] M. Dehn, Papers on Group Theory and Topology, Springer-Verlag, Berlin, 1987. MR 88d:01041

[GeS1] S. M. Gersten and H. Short, Small Cancellation Theory and Automatic Groups, Invent. Math. 102 (1990), 305-334. MR 92c:20058

[GeS2] _ Small Cancellation Theory and Automatic Groups: Part II, Invent. Math. 105 (1991), 641-662. MR 92j:20030

[J1] K. Johnsgard, The Structure of the Cayley Complex and a Cubic-time Algorithm for Solving the Conjugacy Problem for Groups of Prime Alternating Knots, Univ. IllinoisUrbana-Champaign: Ph. D. thesis, 1993.

[J2] Geometric Tilings for Equal Geodesic Words in $C^{\prime \prime}(p)-T(q)$ Group Presentations, 1993, Preprint.

[K] I. Kapovitch, Small Cancellation Groups and Translation Numbers, 1993, Preprint.

[LS] R. C. Lyndon and P. E. Schupp, Combinatorial Group Theory, Springer-Verlag, Berlin, 1977. MR 58:28182

[M] W. Menasco, Closed Incompressible Surfaces in Alternating Knot and Link Complements, Topology 23 (1984), 37-44. MR 86b:57004

[P] C. D. Papakyriakopoulos, On Dehn's Lemma and the Asphericity of Knots, Ann. of Math. 66 (1957), 1-26. MR 19:761a

[Scb] H. Schubert, Die eindeutige Zerlegbarkeit eines Knotens in Primknoten, Sitzungsber. Heidelberger Akad. Wiss. Math.-Natur. Kl. 1949 (3), 57-104. MR 11:196f

[Scp] P. E. Schupp, On Dehn's Algorithm and the Conjugacy Problem, Math. Ann. 178 (1968), 119-130. MR 38:5901

[Se] Z. Sela, The Conjugacy Problem for Knot Groups, Topology 32 (1993), 363-369. MR 94g:57012

[Wa] F. Waldhausen, The Word Problem in Fundamental Groups of Sufficiently Large Irreducible 3-Manifolds, Ann. of Math. 88 (1968), 272-280. MR 39:2167

[We] C. Weinbaum, The Word and Conjugacy Problem for the Knot Group of any Tame Prime Alternating Knot, Proc. Amer. Math. Soc. 22 (1971), 22-26. MR 43:4895

Department of Mathematics, Cornell University, Ithaca, New York 14853

E-mail address: karinj@math.cornell.edu 